\title{
A Screening of UNF Targets Identifies $R n b$, a Novel Regulator of Drosophila Circadian Rhythms
}

\author{
Anatoly Kozlov, ${ }^{1 \star}$ Edouard Jaumouillé, ${ }^{1 \star}$ Pedro Machado Almeida, ${ }^{1}$ Rafael Koch, ${ }^{1}$ Joseph Rodriguez, ${ }^{2}$ \\ - Katharine C. Abruzzi, ${ }^{2}$ and ${ }^{\circledR}$ Emi Nagoshi ${ }^{1}$ \\ ${ }^{1}$ Department of Genetics and Evolution, Sciences III, University of Geneva, Geneva-4, CH-1211, Switzerland, and ${ }^{2}$ Howard Hughes Medical Institute, \\ National Center for Behavioral Genomics, Department of Biology, Brandeis University, Waltham, Massachusetts 02451
}

Behavioral circadian rhythms are controlled by multioscillator networks comprising functionally different subgroups of clock neurons. Studies have demonstrated that molecular clocks in the fruit fly Drosophila melanogaster are regulated differently in clock neuron subclasses to support their specific functions (Lee et al., 2016; Top et al., 2016). The nuclear receptor unfulfilled (unf) represents a regulatory node that provides the small ventral lateral neurons (s-LNvs) unique characteristics as the master pacemaker (Beuchle et al., 2012). We previously showed that UNF interacts with the s-LNv molecular clocks by regulating transcription of the core clock gene period (per) (Jaumouillé et al., 2015). To gain more insight into the mechanisms by which UNF contributes to the functioning of the circadian master pacemaker, we identified UNF target genes using chromatin immunoprecipitation. Our data demonstrate that a previously uncharacterized gene $C G 7837$, which we termed $R$ and $B(R n b)$, acts downstream of UNF to regulate the function of the s-LNvs as the master circadian pacemaker. Mutations and LNv-targeted adult-restricted knockdown of Rnb impair locomotor rhythms. RNB localizes to the nucleus, and its loss-of-function blunts the molecular rhythms and output rhythms of the s-LNvs, particularly the circadian rhythms in PDF accumulation and axonal arbor remodeling. These results establish a second pathway by which UNF interacts with the molecular clocks in the s-LNvs and highlight the mechanistic differences in the molecular clockwork within the pacemaker circuit.

Key words: behavior; chromatin immunoprecipitation; circadian rhythms; Drosophila; molecular mechanism; pacemaker neurons

Significance Statement

Circadian behavior is generated by a pacemaker circuit comprising diverse classes of pacemaker neurons, each of which contains a molecular clock. In addition to the anatomical and functional diversity, recent studies have shown the mechanistic differences in the molecular clockwork among the pacemaker neurons in Drosophila. Here, we identified the molecular characteristics distinguishing the s-LNvs, the master pacemaker of the locomotor rhythms, from other clock neuron subtypes. We demonstrated that a newly identified gene $R n b$ is an s-LNv-specific regulator of the molecular clock and essential for the generation of circadian locomotor behavior. Our results provide additional evidence to the emerging view that the differential regulation of the molecular clocks underlies the functional differences among the pacemaker neuron subgroups.

\section{Introduction}

Circadian rhythms enable organisms to anticipate and adapt to rhythmic daily and seasonal environmental changes. The molec-

\footnotetext{
Received Oct. 24, 2016; revised May 12, 2017; accepted May 13, 2017.

Author contributions: A.K., E.J., and E.N. designed research;A.K., E.J., P.M.A., R.K., and K.C.A. performed research; J.R. contributed unpublished reagents/analytic tools; A.K., E.J.,P.M.A., R.K., J.R., K.C.A., and E.N. analyzed data; A.K., R.K., and E.N. wrote the paper.

This work was supported by the JST PREST0 program, Swiss National Science Foundation 31003A_149893, European Research Council ERC-StG-311194, the Georges \& Antoine Claraz Donation, and the University of Geneva. We thank the Bloomington Drosophila Stock Center (NIH P400D018537) and the Vienna Drosophila Resource Center for providing the fly stocks; Tzumin Lee for providing the anti-UNF antibodies; the Berkeley Drosophila Genome Project for a cDNA clone; BestGene Inc for the injection service; Michael Rosbash for generously sharing the ChIP protocol and providing technical support; M. Fernanda Ceriani and Ueli Schibler for helpful comments on the manuscript; and Ken Sugino for assistance with the bioinformatics analysis.

The authors declare no competing financial interests.
}

ular clocks, which largely rely on transcriptional/translational feedback loops (TTFLs), generate $24 \mathrm{~h}$ rhythms in eukaryotes (Mohawk et al., 2012; Hardin and Panda, 2013). In Drosophila, the periodic transcription of period (per) and timeless (tim) by the CLOCK (CLK)/CYCLE (CYC) heterodimer and feedback inhibition of CLK/CYC-dependent transcription by the PER/TIM complex constitute the principal TTFL of the molecular clock.

\footnotetext{
*A.K. and E. J. contributed equally to this work.

J. Rodriguez' present address: Laboratory of Receptor Biology and Gene Expression, National Cancer Institute, National Institutes of Health, 41 Center Drive, Bethesda, MD 20892.

Correspondence should be addressed to Dr. Emi Nagoshi, Department of Genetics and Evolution, Sciences III, University of Geneva, 30 Quai Ernest-Ansermet, Geneva-4, CH-1211, Switzerland. E-mail: Emi.Nagoshi@unige.ch. DOI:10.1523/JNEUROSCI.3286-16.2017

Copyright $\odot 2017$ the authors $\quad 0270-6474 / 17 / 376673-13 \$ 15.00 / 0$
} 
A
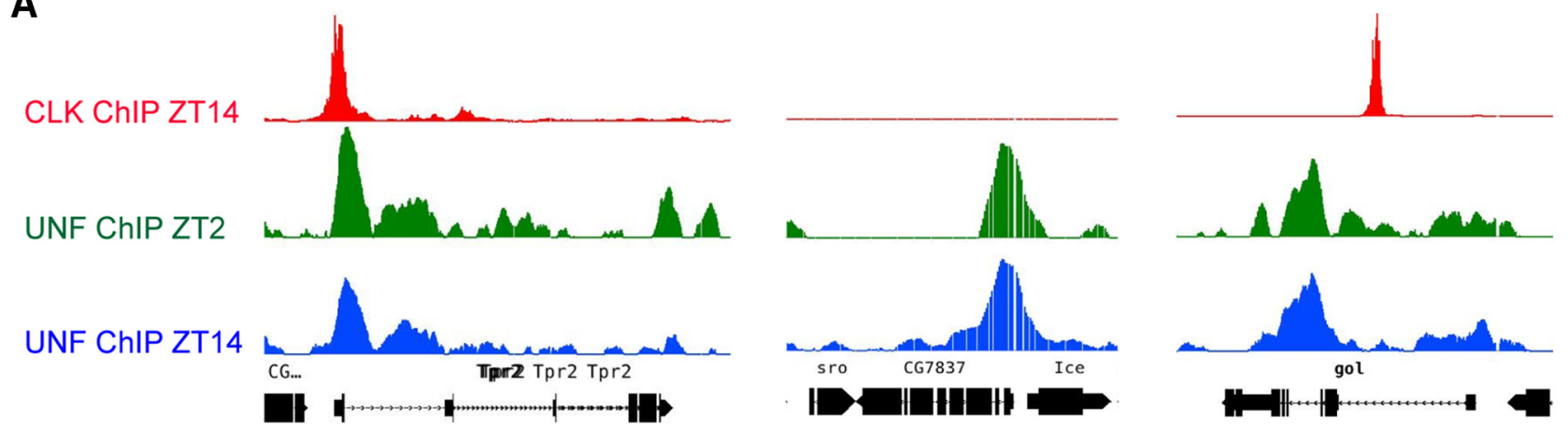

B
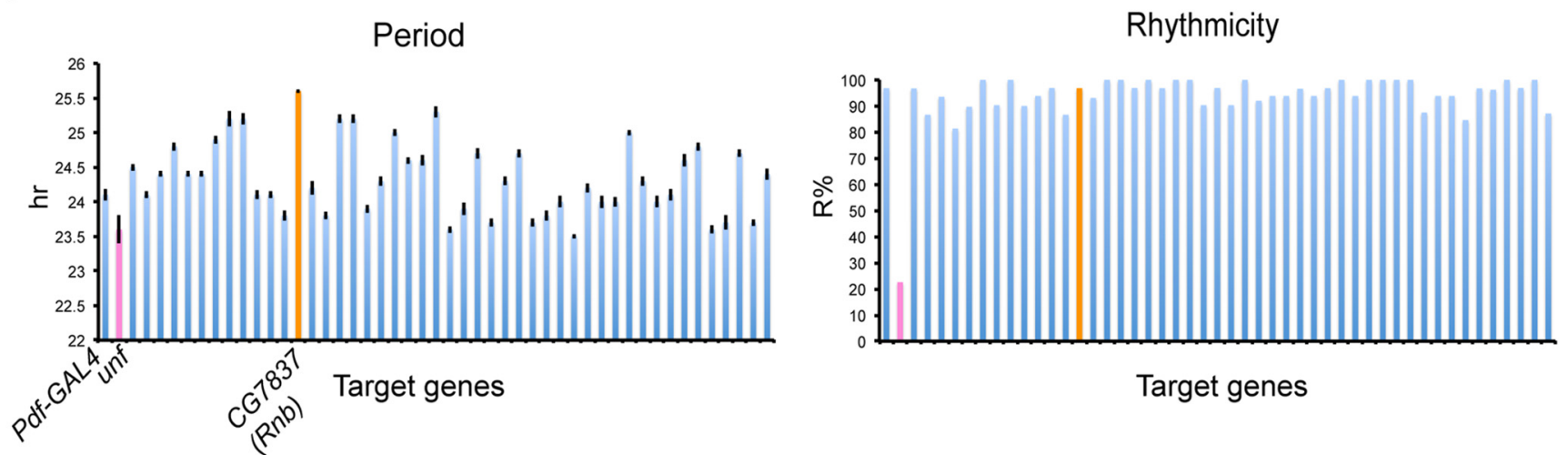

C

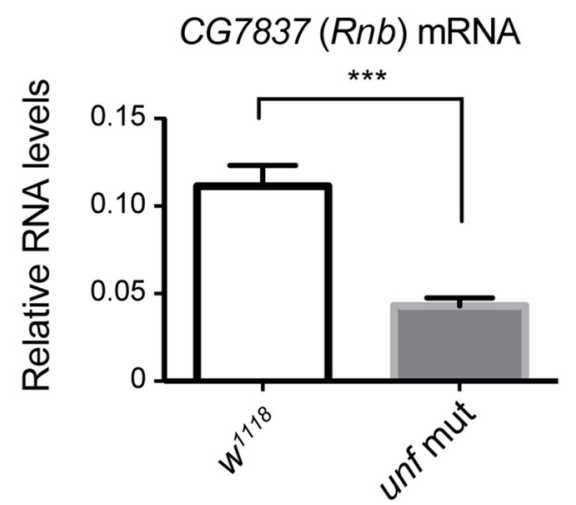

D

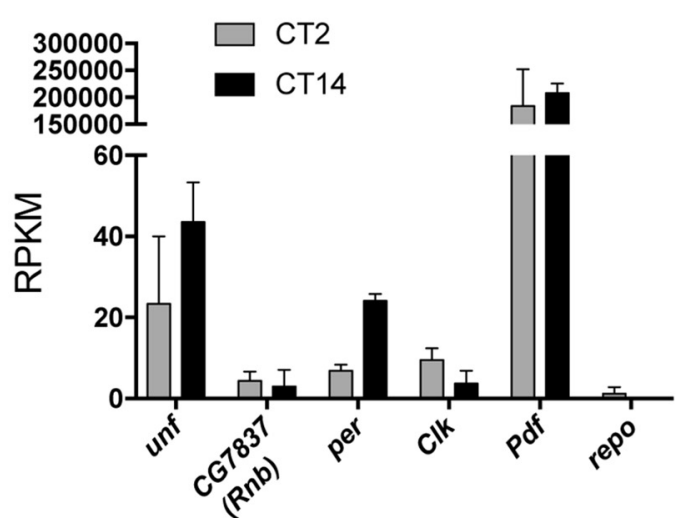

Figure 1. CG7837 (Rnb) is a direct target of UNF. A, Binding of CLK and UNF on the Tpr2, CG7837 (Rnb), and gol genes is shown as examples of the ChIP results. CLK binding data were obtained from Abruzzi et al. (2011). B, UNF target genes were knocked down by RNAi using Pdf-GAL4 and tested for locomotor behavior. The RNAi lines listed in Table 2 from top to bottom correspond to the $x$-axis from left to right. C, Relative Rnb mRNA levels normalized to the Ef1 $\beta$ mRNA levels were analyzed using qPCR in the heads of $w^{1118}$ and unf ${ }^{\alpha 1 / 20001}$ mutant flies. Rnb levels were significantly reduced in the unf ${ }^{\alpha 1 / 20001}$ flies. ${ }^{* * *} p<0.001$ (Student's $t$ test). D, RNAseq of the LNvs isolated at two time points in DD3 revealed the expression of Rnb transcripts in the LNvs. Data represent the average RPKM (reads per kilobase of transcript per million mapped reads) of two independent samples \pm SD. The transcripts of unf, Pdf, and clock genes, but not the glia-specific gene reverse polarity (repo), were identified, reflecting the LNv specificity of the sequenced libraries.

CLK/CYC also activates transcription of $p d p-1$ and vrille (vri), and PDP-1 and VRI proteins regulate transcription of Clk, thereby forming a stabilizing loop (Hardin, 2011). In addition, post-transcriptional and post-translational regulation play important roles in generating $24 \mathrm{~h}$ rhythms (Lim and Allada, 2013).

Drosophila circadian locomotor behavior is organized by a neural circuit composed of anatomically and functionally diverse groups of clock-containing neurons. The morning and evening activity bouts under light-dark (LD) cycles are controlled by the small ventral lateral neurons (s-LNvs; M-oscillator) and the E-oscillator group, including the dorsal lateral neurons (LNds) and the fifth s-LNv (Peschel and Helfrich-Forster, 2011). Freerunning behavioral rhythms in constant darkness (DD) are pre- dominantly driven by the $\mathrm{M}$-oscillator at least within a certain range of periods (Stoleru et al., 2005; Yao and Shafer, 2014). A subgroup of dorsal neurons, DN1s, relay output signals from the $\mathrm{M}$-oscillator to locomotor output circuit to generate morning activity peaks and contribute to the free-running locomotor rhythms (Cavanaugh et al., 2014). DN1s also integrate environmental information and modulate the operation of $\mathrm{M}$ - and E-oscillators in driving morning and evening peaks (Zhang et al., 2010a, b).

Gene expression differences among clock neurons underlie their functional differences (Kula-Eversole et al., 2010; Nagoshi et al., 2010). Most notably, the neuropeptide pigment-dispersing factor (PDF), which is expressed only in the s- and l-LNvs, is 
Table 1. UNF binding peaks ${ }^{a}$

\begin{tabular}{|c|c|c|c|c|c|c|c|c|}
\hline Chromatin & Start & End & Annotation & ZT14 rank & ZT14p & ZT2 rank & ZT2p & CLKBD \\
\hline$x$ & 8036801 & 8040825 & $\begin{array}{l}\text { Smox-promoter, CG17982-promoter, CG2263-promoter, } \\
\text { CG17982-exon, CG15336-promoter, CG2129-exon, } \\
\text { CG2129-promoter, CG2263-exon }\end{array}$ & 1 & $3.3 \mathrm{E}-29$ & 18 & $3.3 \mathrm{E}-11$ & \\
\hline $3 R$ & 20075140 & 20079908 & $\begin{array}{l}\text { mask-exon, CG5706-promoter, jar-intron, jar-exon, } \\
\text { CG5706-intron, CG5706-exon }\end{array}$ & 2 & $3.3 \mathrm{E}-25$ & & & \\
\hline $3 R$ & 3299277 & 3301524 & $\begin{array}{l}\text { CG1142-exon, snRNA:7SK-promoter, snRNA:7SK-exon, } \\
\text { CG1142-promoter, snoRNA:MeU5-C46-promoter }\end{array}$ & 3 & $9.7 \mathrm{E}-25$ & & & \\
\hline $2 \mathrm{~L}$ & 16491360 & 16493309 & Tpr2-exon, Tpr2-promoter, Tpr2-intron & 4 & $9.0 \mathrm{E}-24$ & 4 & $2.8 \mathrm{E}-15$ & Tpr2 \\
\hline $2 \mathrm{R}$ & 18958854 & 18960385 & I(2)k09913-exon & 5 & $1.4 \mathrm{E}-23$ & 11 & $3.6 \mathrm{E}-12$ & \\
\hline $2 \mathrm{R}$ & 9317001 & 9320635 & $\begin{array}{l}\text { Ser8-promoter, CG30065-exon, CG42321-exon, } \\
\text { CG42321-intron, CG30065-promoter, CG42321- } \\
\text { promoter }\end{array}$ & 6 & $5.5 \mathrm{E}-21$ & & & \\
\hline $3 R$ & 25623780 & 25625727 & $\begin{array}{l}\text { CG7837-exon, Ice-exon, CG7837-intron, Ice-promoter, } \\
\text { CG7837-promoter }\end{array}$ & 7 & $8.0 \mathrm{E}-20$ & 3 & $6.2 \mathrm{E}-17$ & \\
\hline $2 R$ & 15301421 & 15303508 & snoRNA:hts-a-exon, snoRNA:hts-a-promoter, hts-intron & 8 & $2.9 \mathrm{E}-18$ & 6 & $1.3 \mathrm{E}-13$ & \\
\hline $2 \mathrm{R}$ & 9317424 & 9320594 & $\begin{array}{l}\text { Ser8-promoter, CG42321-intron, CG30065-promoter, } \\
\text { CG42321-promoter }\end{array}$ & & & 1 & $2.8 \mathrm{E}-17$ & \\
\hline $3 R$ & 3299796 & 3301424 & $\begin{array}{l}\text { snRNA:7SK-promoter, snRNA:7SK-exon, CG1142- } \\
\text { promoter, snoRNA:MeU5-C46-promoter }\end{array}$ & & & 2 & $4.2 \mathrm{E}-17$ & \\
\hline $2 \mathrm{R}$ & 20962653 & 20965558 & gol-intron, gol-exon & 9 & $1.2 \mathrm{E}-16$ & 8 & $1.1 \mathrm{E}-12$ & gol \\
\hline $3 R$ & 4493289 & 4495665 & CG8036-exon, CG8036-intron, CG8036-promoter & 10 & $1.7 \mathrm{E}-16$ & 19 & $4.5 \mathrm{E}-11$ & \\
\hline $2 R$ & 2058366 & 2060021 & EcR-promoter, Cyp6w1-promoter & 11 & $2.6 \mathrm{E}-16$ & 7 & $9.0 \mathrm{E}-13$ & $\mathrm{ECR}$ \\
\hline $3 \mathrm{~L}$ & 14666572 & 14668637 & $\begin{array}{l}\text { CG43121-promoter, CG4914-intron, ome-intron, } \\
\text { CG4914-exon }\end{array}$ & 12 & 4.1E-16 & & & \\
\hline $3 \mathrm{R}$ & 8862579 & 8864238 & $\begin{array}{l}\text { CG11668-exon, CG11668-intron, CG11668-promoter, } \\
\text { ry-intron, ry-exon }\end{array}$ & 13 & $1.1 \mathrm{E}-15$ & & & \\
\hline $3 R$ & 18619187 & 18620688 & CG7029-exon & 14 & $1.8 \mathrm{E}-15$ & 5 & $5.8 \mathrm{E}-14$ & \\
\hline $2 R$ & 13278911 & 13280451 & Sip1-promoter & 15 & $2.3 \mathrm{E}-15$ & 16 & $1.4 \mathrm{E}-11$ & \\
\hline $2 \mathrm{~L}$ & 20873588 & 20875696 & sky-exon, sky-intron & 16 & $4.4 \mathrm{E}-15$ & & & \\
\hline$x$ & 2587801 & 2588943 & CG2650-exon, CG2658-exon, CG2650-promoter & 17 & $4.7 \mathrm{E}-15$ & & & \\
\hline $2 R$ & 17269291 & 17271559 & CG10795-exon, CG10795-intron & 18 & $5.6 \mathrm{E}-14$ & & & \\
\hline $3 R$ & 22772208 & 22774253 & $\begin{array}{l}\text { CG14252-exon, CG18472-intron, CG18472-exon, } \\
\text { CG18472-promoter, CG14252-intron }\end{array}$ & 19 & $7.3 \mathrm{E}-14$ & 40 & $6.3 \mathrm{E}-08$ & \\
\hline $3 R$ & 13444545 & 13446800 & $\begin{array}{l}\text { CG14324-promoter, CG14326-promoter, CG14326-exon, } \\
\text { CG14326-intron }\end{array}$ & 20 & $3.4 \mathrm{E}-13$ & & & \\
\hline $3 \mathrm{~L}$ & 5789797 & 5791159 & Tektin-C-promoter & 21 & $4.7 \mathrm{E}-13$ & 39 & $6.2 \mathrm{E}-08$ & \\
\hline $3 \mathrm{~L}$ & 18610853 & 18612096 & $\begin{array}{l}\text { CG13380-promoter, CG13380-exon, CG4174-exon, } \\
\text { not-exon, CG4174-promoter }\end{array}$ & 22 & $6.4 \mathrm{E}-13$ & 23 & $2.3 \mathrm{E}-09$ & \\
\hline$x$ & 5417756 & 5419200 & $\begin{array}{l}\text { snoRNA:Psi28S-3342-promoter, snoRNA:Psi28S-3342- } \\
\text { exon }\end{array}$ & 23 & $1.4 \mathrm{E}-12$ & 9 & $1.7 \mathrm{E}-12$ & \\
\hline $3 \mathrm{~L}$ & 441077 & 442721 & klar-intron, klar-exon, klar-promoter & 24 & $2.4 \mathrm{E}-12$ & & & \\
\hline $3 \mathrm{~L}$ & 3095106 & 3097041 & Cht7-intron & 25 & $4.1 \mathrm{E}-12$ & 10 & $2.5 \mathrm{E}-12$ & \\
\hline $3 R$ & 13443098 & 13444388 & CG14326-promoter, CG14324-exon & & & 13 & $9.3 \mathrm{E}-12$ & \\
\hline$x$ & 1676738 & 1677935 & Adar-exon, Adar-intron, CG42666-intron & & & 14 & $9.8 \mathrm{E}-12$ & \\
\hline $3 R$ & 20076127 & 20078482 & CG5706-promoter, CG5706-intron, CG5706-exon & & & 15 & $1.2 \mathrm{E}-11$ & \\
\hline $3 R$ & 5039548 & 5040831 & pum-intron, pum-exon & 26 & $1.4 \mathrm{E}-11$ & 12 & $5.2 \mathrm{E}-12$ & \\
\hline $3 \mathrm{~L}$ & 14666655 & 14668094 & CG43121-promoter, ome-intron, CG4914-exon & & & 17 & $1.5 \mathrm{E}-11$ & \\
\hline $2 \mathrm{R}$ & 12106837 & 12109098 & mrj-intron & 27 & $1.5 \mathrm{E}-11$ & 29 & $8.4 \mathrm{E}-09$ & \\
\hline $2 R$ & 4555166 & 4557028 & Acsl-intron, Acsl-promoter & 28 & $1.9 \mathrm{E}-11$ & & & \\
\hline $3 R$ & 19555340 & 19556770 & $\begin{array}{l}\text { CG10365-exon, RNaseMRP:RNA-exon, CG10365-intron, } \\
\text { RNaseMRP:RNA-promoter }\end{array}$ & 29 & $2.2 \mathrm{E}-11$ & 70 & $2.7 \mathrm{E}-06$ & \\
\hline $2 \mathrm{~L}$ & 3172537 & 3173744 & $\begin{array}{l}\text { CG34406-exon, CG31698-intron, CG31698-exon, } \\
\text { CG34406-intron, CG15404-promoter, CG34406- } \\
\text { promoter }\end{array}$ & 30 & $3.2 \mathrm{E}-11$ & 30 & $8.7 \mathrm{E}-09$ & \\
\hline $3 \mathrm{~L}$ & 22839989 & 22841597 & CR43426-promoter & 31 & $3.4 \mathrm{E}-11$ & & & \\
\hline $2 \mathrm{R}$ & 7539150 & 7540664 & $\begin{array}{l}\text { CG34228-intron, Roc2-intron, Roc2-exon, CG34228- } \\
\text { exon, CG34228-promoter, CG9003-promoter, } \\
\text { CG13198-promoter }\end{array}$ & 32 & 4.1E-11 & & & Roc2 \\
\hline $3 R$ & 13442719 & 13444544 & $\begin{array}{l}\text { CG14324-intron, CG14324-promoter, CG14326- } \\
\text { promoter, CG14324-exon }\end{array}$ & 33 & $6.2 \mathrm{E}-11$ & & & \\
\hline $2 \mathrm{R}$ & 13491220 & 13493358 & lack-exon, CG10936-exon, CG10936-intron & 34 & $8.0 \mathrm{E}-11$ & & & \\
\hline $2 \mathrm{R}$ & 14664507 & 14665831 & CG15093-promoter & 35 & $1.8 \mathrm{E}-10$ & 34 & $\begin{array}{r}2.6 \mathrm{E}-08 \\
(T t\end{array}$ & $\begin{array}{r}\text { (G15093 } \\
\text { continued) }\end{array}$ \\
\hline
\end{tabular}


Table 1. Continued

\begin{tabular}{|c|c|c|c|c|c|c|c|c|}
\hline Chromatin & Start & End & Annotation & ZT14 rank & ZT14p & ZT2 rank & ZT2p & CLK BD \\
\hline $3 R$ & 2954340 & 2957636 & $\begin{array}{l}\text { CG31493-promoter, CR42875-intron, CR42875-exon, } \\
\text { CG31493-intron, CG31248-intron, CG31493-exon, } \\
\text { CG10068-promoter, CG31248-exon, CG31248- } \\
\text { promoter }\end{array}$ & 36 & $4.6 \mathrm{E}-10$ & & & \\
\hline $2 \mathrm{R}$ & 11747802 & 11749162 & Gpo-1-exon, Gpo-1-intron, Gpo-1-promoter & 37 & $6.7 \mathrm{E}-10$ & & & Gpo-1 \\
\hline$x$ & 8105175 & 8106920 & sdt-intron & 51 & $1.2 \mathrm{E}-08$ & 20 & $6.8 \mathrm{E}-10$ & sdt \\
\hline $3 R$ & 17490794 & 17492282 & $\begin{array}{l}\text { DNApol-alpha180-exon, CG15497-exon, CG15497- } \\
\text { promoter, CG15497-intron }\end{array}$ & 38 & $7.0 \mathrm{E}-10$ & & & \\
\hline $2 R$ & 5480924 & 5482611 & $\begin{array}{l}\text { CG1902-exon, clos-exon, CG30338-intron, clos-intron, } \\
\text { CG1902-promoter, CG30338-exon, CG1902-intron, } \\
\text { CG30338-promoter }\end{array}$ & 39 & $7.1 \mathrm{E}-10$ & & & \\
\hline $3 \mathrm{~L}$ & 17393439 & 17394974 & noe-promoter, blot-exon, noe-exon, blot-intron & 40 & $8.1 \mathrm{E}-10$ & & & \\
\hline $2 R$ & 2039462 & 2040973 & EcR-exon, EcR-intron & 41 & $1.1 \mathrm{E}-09$ & 26 & $2.9 \mathrm{E}-09$ & $\mathrm{EcR}$ \\
\hline $3 R$ & 2644498 & 2646006 & 7SLRNA:CR42652-exon, 7SLRNA:CR32864-exon & 45 & $4.2 \mathrm{E}-09$ & 21 & $1.1 \mathrm{E}-09$ & \\
\hline $3 \mathrm{~L}$ & 17393439 & 17394858 & noe-promoter, noe-exon, blot-exon, blot-intron & & & 22 & $1.4 \mathrm{E}-09$ & \\
\hline $3 R$ & 5154054 & 5155329 & $\begin{array}{l}\text { CG8861-exon, CG8861-intron, CG12951-exon, CG12951- } \\
\text { intron, CG8861-promoter }\end{array}$ & 42 & 2.1E-09 & & & \\
\hline $3 \mathrm{~L}$ & 19807399 & 19808572 & cyc-intron, cyc-exon, Сyp305a1-promoter & 65 & $8.2 \mathrm{E}-08$ & 24 & $2.4 \mathrm{E}-09$ & \\
\hline $2 R$ & 13503641 & 13504878 & $\begin{array}{l}\text { snoRNA:Or-aca4-exon, CG10936-intron, snoRNA: } \\
\text { Or-aca4-promoter }\end{array}$ & & & 25 & $2.5 \mathrm{E}-09$ & \\
\hline $2 \mathrm{~L}$ & 13892279 & 13893601 & cenG1A-promoter, cenG1A-intron & 43 & $2.5 \mathrm{E}-09$ & & & cenG1A \\
\hline $2 \mathrm{R}$ & 8221206 & 8224776 & $\begin{array}{l}\text { CG34021-intron, Den1-promoter, CG8490-exon, } \\
\text { garz-exon, CG34021-promoter, CG8490-intron, } \\
\text { CG34021-exon, CG8490-promoter }\end{array}$ & 44 & 2.7E-09 & & & \\
\hline
\end{tabular}

aUNF binding peaks analyzed using anti-UNF ChIP-chip assays are ordered by rank. CLK BD, CLK target gene.

essential for the role of the M-oscillator in generating morning activity peak and for driving free-running behavior (Renn et al., 1999). Recent studies also revealed that TTFL is regulated differently in clock neuron subtypes, suggesting the presence of regulatory mechanisms specific for the s-LNv master pacemaker neurons (Lee et al., 2016; Top et al., 2016). Consistently, the unfulfilled (unf; DHR51) gene encoding a nuclear receptor is selectively expressed in the LNvs within the clock neuron subgroups and is necessary for the functioning of the s-LNvs as the master pacemaker (Beuchle et al., 2012). UNF acts together with the second nuclear receptor E75, a homolog of mammalian REVERB $\alpha / \beta$, to enhance per transcription, thereby reinforcing the master pacemaker TTFL (Jaumouillé et al., 2015).

To better understand the mechanisms by which UNF contributes to the functioning of the M-oscillator, here we identify UNF direct targets using chromatin immunoprecipitation (ChIP). The behavioral screening of UNF target genes in conjunction with functional assays reveals that a previously uncharacterized gene CG7837, which we name Rnb, acts downstream of UNF to regulate the molecular clockwork of the s-LNvs. By demonstrating the second pathway through which UNF regulates molecular clockwork specifically in the s-LNvs, these results highlight the unexpected diversity of the molecular clock makeup among circadian pacemaker neurons.

\section{Materials and Methods}

Fly rearing, crosses, and strains. Drosophila melanogaster were reared on standard cornmeal/agar medium supplemented with yeast in $12 \mathrm{~h} / 12 \mathrm{~h}$ $\mathrm{LD}$ cycles at $25^{\circ} \mathrm{C}$, unless otherwise mentioned. UAS-RNAi lines were obtained from the Vienna Drosophila Resource Center (VDRC). D52HGAL4 (Aso et al., 2009), unf $f^{x 1}$, and unf ${ }^{20001}$ (Beuchle et al., 2012) were previously described. The following lines were obtained from the Bloomington Drosophila Stock Center: CG7837 $7^{f 04616}, C G 7837^{f 05400}$ and $w^{1118}$; $D f(3 R) B S C 547$ (dubbed $D f$ ).

Conditional knockdown or overexpression experiments were performed by combining GAL4 (Pdf-GAL4 or gal1118) and tubulin-GAL80 $0^{\text {ts }}$ with the UAS-transgene and shifting the temperature of fly rearing be- tween $18^{\circ} \mathrm{C}$ (permissive temperature for GAL80 ${ }^{\mathrm{ts}}$ ) and $29^{\circ} \mathrm{C}$ (restrictive temperature for GAL80 ${ }^{\text {ts }}$ ) (McGuire et al., 2004). To express UAStransgenes only during development, the flies were crossed and reared at $29^{\circ} \mathrm{C}$ until eclosion, and the emerged flies were immediately transferred to and maintained at $18^{\circ} \mathrm{C}$ for the following experiments. To express UAS-transgenes only during adulthood, the flies were crossed and reared at $18^{\circ} \mathrm{C}$, and the emerged flies were immediately transferred to $29^{\circ} \mathrm{C}$.

To generate the UAS-Rnb::Venus construct, the Rnb coding sequence without the stop codon was PCR-amplified from the LD3331 clone (Berkeley Drosophila Genome Project) and cloned into the pBIDUASC-GV vector (Wang et al., 2012) using the Gateway Cloning System (Invitrogen). UAS-Rnb::Venus construct was integrated into the attP40 landing site on the second chromosome by phiC31-mediated sitedirected transgenesis. Embryo injections were conducted at BestGene.

Behavioral assays. The locomotor behavior was conducted as previously described (Beuchle et al., 2012) and analyzed using FaasX software (Blanchardon et al., 2001). Briefly, male flies were first entrained in 12 $\mathrm{h} / 12 \mathrm{~h} \mathrm{LD}$ cycles for $4 \mathrm{~d}$, followed by the recording in DD for $8-10 \mathrm{~d}$. The flies with a power $>20$ and a width $>2.5$ according to the $\chi^{2}$ periodogram analysis were defined as rhythmic. The significance threshold was set to $5 \%$. The $\chi^{2}$ test was used to compare the rhythmicity of the flies, and the Student's $t$ test (two-tailed) was used to compare the freerunning period.

Immunostaining, microscopy, and quantification. The immunostaining of adult fly brains with anti-PER and anti-PDF antibodies was performed as previously described (Shafer et al., 2002). RNB::VENUS and mCD8:: VENUS were immunostained using anti-GFP antibodies (Invitrogen rabbit anti-GFP \#6455) in a dilution 1:500. Briefly, the dissected brains were fixed in $4 \%$ PFA at room temperature for $15 \mathrm{~min}$, followed by washing three times for $10 \mathrm{~min}$ in PBT (PBS, 0.5\% Triton X-100). Antibodies were diluted in PBT, and primary antibody staining was performed overnight.

Images were captured using a Leica SP5 confocal microscope. A minimum of 10 brain hemispheres was subjected to quantification using ImageJ software (National Institutes of Health). Anti-PER signal was quantified as previously described (Beuchle et al., 2012). PDF immunoreactivity in the s-LNv cell bodies and axonal termini was quantified from the sum projections of the confocal $Z$ stacks. The region of interest (ROI) for the axonal termini (from the tip of the s-LNv dorsal termini until 
Table 2. Free-running locomotor rhythms of the flies expressing RNAs against UNF targets in the LNvs ${ }^{a}$

\begin{tabular}{|c|c|c|c|c|c|c|c|}
\hline Gene & Rank & VDRC & ID & $\% R$ & Period \pm SEM (h) & Power \pm SEM & $\bar{n}$ \\
\hline Pdf-GAL4I+ & - & - & - & 96.8 & $24.1 \pm 0.08$ & $126.1 \pm 6.63$ & 31 \\
\hline Pdf $>$ miR-unf & - & - & 一 & 22.6 & $23.6 \pm 0.20$ & $47.9 \pm 6.52$ & 31 \\
\hline CG15336 & 1 & 102385 & KK & 96.7 & $24.5 \pm 0.05$ & $133.2 \pm 5.86$ & 29 \\
\hline CG17982 & 1 & 33035 & GD & 86.7 & $24.1 \pm 0.05$ & $118.1 \pm 5.58$ & 31 \\
\hline CG2262 & 1 & 105687 & KK & 93.5 & $24.4 \pm 0.04$ & $125.1 \pm 4.64$ & 31 \\
\hline CG2263 & 1 & 33515 & GD & 81.3 & $24.8 \pm 0.06$ & $122.7 \pm 5.29$ & 28 \\
\hline CG33106 & 2 & 103411 & KK & 89.7 & $24.4 \pm 0.04$ & $122.8 \pm 4.96$ & 31 \\
\hline CG5695 & 2 & 108221 & KK & 100 & $24.4 \pm 0.04$ & $133.5 \pm 4.18$ & 31 \\
\hline CG5706 & 2 & 42046 & GD & 90.3 & $24.9 \pm 0.06$ & $118.4 \pm 6.19$ & 28 \\
\hline CG4599 & 4 & 107813 & KK & 100 & $25.2 \pm 0.11$ & $110.9 \pm 6.51$ & 31 \\
\hline CG3082 & 5 & 102477 & KK & 90 & $25.2 \pm 0.08$ & $120.4 \pm 6.03$ & 32 \\
\hline CG30065 & 6 & 102347 & KK & 93.8 & $24.1 \pm 0.06$ & $123.3 \pm 6.19$ & 10 \\
\hline CG4812 & 6 & 104534 & KK & 96.9 & $24.1 \pm 0.05$ & $134.4 \pm 3.87$ & 15 \\
\hline CG42321 & 6 & 107000 & KK & 86.7 & $23.8 \pm 0.07$ & $98.3 \pm 5.98$ & 30 \\
\hline CG7837 & 7 & 23099 & GD & 96.8 & $25.6 \pm 0.03$ & $139.8 \pm 4.88$ & 32 \\
\hline CG7788 & 7 & 28065 & GD & 93.1 & $24.2 \pm 0.10$ & $86.4 \pm 5.54$ & 29 \\
\hline CG9325 & 8 & 29102 & GD & 100 & $23.8 \pm 0.05$ & $180.0 \pm 2.93$ & 32 \\
\hline CG8036 & 9 & 105633 & KK & 100 & $25.2 \pm 0.06$ & $130.1 \pm 6.50$ & 31 \\
\hline CG2679 & 12 & 107822 & KK & 96.9 & $25.2 \pm 0.06$ & $132.3 \pm 5.22$ & 32 \\
\hline CG1765 & 14 & 37059 & GD & 100 & $23.9 \pm 0.06$ & $190.0 \pm 4.31$ & 31 \\
\hline CG42280 & 15 & 8369 & $G D$ & 96.8 & $24.3 \pm 0.07$ & $115.4 \pm 6.33$ & 26 \\
\hline CG11668 & 16 & 105073 & KK & 100 & $25.0 \pm 0.05$ & $135.9 \pm 5.00$ & 31 \\
\hline CG7029 & 17 & 107658 & KK & 100 & $24.6 \pm 0.05$ & $152.7 \pm 6.51$ & 31 \\
\hline CG9339 & 20 & 108736 & KK & 90.3 & $24.6 \pm 0.08$ & $90.3 \pm 6.80$ & 30 \\
\hline CG2650 & 21 & 105930 & KK & 96.9 & $25.3 \pm 0.08$ & $113.9 \pm 5.73$ & 31 \\
\hline \multirow[t]{2}{*}{ CG10795 } & 22 & 110153 & KK & 90.3 & $23.6 \pm 0.05$ & $102.1 \pm 5.04$ & 31 \\
\hline & & 8833 & $G D$ & 100 & $23.9 \pm 0.09$ & $119.2 \pm 6.30$ & 28 \\
\hline \multirow[t]{2}{*}{ CG14324 } & 26 & 102574 & KK & 92 & $24.7 \pm 0.08$ & $120.6 \pm 4.98$ & 25 \\
\hline & & 5897 & GD & 93.8 & $23.7 \pm 0.06$ & $98.1 \pm 4.31$ & 32 \\
\hline CG9755 & 37 & 45815 & $G D$ & 93.8 & $24.3 \pm 0.06$ & $106.6 \pm 5.26$ & 32 \\
\hline \multirow[t]{2}{*}{ CG12598 } & 39 & 105612 & KK & 96.6 & $24.7 \pm 0.06$ & $107.5 \pm 5.57$ & 29 \\
\hline & & 7764 & GD & 93.8 & $23.7 \pm 0.06$ & $121.4 \pm 7.68$ & 32 \\
\hline \multirow[t]{2}{*}{ CG42666 } & 39 & 103667 & KK & 96.8 & $23.8 \pm 0.07$ & $106.7 \pm 4.77$ & 31 \\
\hline & & 38827 & GD & 100 & $24.0 \pm 0.08$ & $96.0 \pm 6.18$ & 31 \\
\hline \multirow[t]{2}{*}{ CG13198 } & 50 & 104553 & KK & 93.8 & $23.5 \pm 0.03$ & $104.8 \pm 7.46$ & 32 \\
\hline & & 44080 & $G D$ & 100 & $24.2 \pm 0.07$ & $121.5 \pm 3.79$ & 32 \\
\hline CG8998 & 50 & 100629 & GD & 100 & $24.0 \pm 0.09$ & $119.9 \pm 4.87$ & 28 \\
\hline CG9003 & 50 & 23482 & GD & 100 & $24.0 \pm 0.07$ & $110.7 \pm 3.99$ & 32 \\
\hline CG8727 & 66 & 11765 & GD & 100 & $25.0 \pm 0.04$ & $185.4 \pm 3.71$ & 28 \\
\hline \multirow[t]{2}{*}{ CG15404 } & 80 & 102242 & KK & 87.5 & $24.3 \pm 0.07$ & $78.5 \pm 5.03$ & 32 \\
\hline & & 23313 & GD & 93.8 & $24.0 \pm 0.08$ & $101.1 \pm 5.10$ & 32 \\
\hline CG31698 & 80 & 110231 & GD & 93.8 & $24.1 \pm 0.08$ & $119.3 \pm 4.86$ & 32 \\
\hline CG2652 & 83 & 33542 & $G D$ & 84.6 & $24.6 \pm 0.09$ & $93.7 \pm 7.23$ & 30 \\
\hline CG2655 & 83 & 104381 & KK & 96.7 & $24.8 \pm 0.06$ & $126.8 \pm 6.42$ & 30 \\
\hline CG30122 & 84 & 106984 & KK & 96.2 & $23.6 \pm 0.06$ & $107.4 \pm 7.41$ & 26 \\
\hline \multirow[t]{2}{*}{ CG33724 } & 84 & 100994 & KK & 100 & $23.7 \pm 0.10$ & $91.3 \pm 6.06$ & 29 \\
\hline & & 48814 & GD & 96.9 & $24.7 \pm 0.05$ & $98.9 \pm 3.96$ & 32 \\
\hline CG8817 & 108 & 13082 & GD & 100 & $23.7 \pm 0.05$ & $190.0 \pm 2.54$ & 32 \\
\hline
\end{tabular}

a Rank, rank in the UNF ChIP. When the gene is bound to UNF at both ZT2 and ZT14, the higher rank is shown. $n$, Number of flies; \%R, \% of rhythmic flies.

where the terminal arbors first branch) was specified with the polygon selection tool, and the intensity sum within each ROI was measured. To quantify s-LNv axonal spread, LNvs projections were visualized by expressing $\mathrm{mCD} 8:$ :Venus in the LNvs. The ROI for the sLNv axonal termini was determined manually as described above and the area of the ROI was measured.

ChIP-chip and data analysis. $w^{1118}$ flies were entrained in LD for $3 \mathrm{~d}$ and collected at ZT2 and ZT14. ChIP using rabbit anti-UNF antibodies (Lin et al., 2009) were performed and hybridized to the GeneChip Drosophila Tiling 2.0R Arrays (Affymetrix) as previously described (Menet et al., 2010). Two independent ChIP-chip experiments were performed at both time points. To assess the UNF ChIP signals outside MB, a ChIPchip assay was performed with the D52H-Gal4, UAS-miR UNF flies (Aso et al., 2009) harvested at ZT2. ChIP-chip data analysis was performed as previously described (Abruzzi et al., 2011) using the MAT algorithm
(Johnson et al., 2006). The ChIP peaks were visualized using the Integrated Genome Browser.

RNA analysis. Total RNA was isolated from adult fly heads using Trizol (Invitrogen) following the manufacturer's instructions. The RNA was reverse-transcribed using oligo $(\mathrm{dT})$ primers, and the resulting $\mathrm{cDNAs}$ were quantified using real-time qPCR as previously described (Nagoshi et al., 2010). The mRNA levels of Rnb were normalized to those of elongation factor $1 \beta$ (Ef1 $\beta$ ).

Cell sorting and RNA sequencing (RNAseq). Manual cell sorting protocol was modified from the previously described method (Nagoshi et al., 2010; Abruzzi et al., 2015). Flies carrying Pdf-GAL4 and tubulin-GAL80 $0^{\text {ts }}$ were combined with UAS-nls::GFP to mark s-LNvs and 1-LNvs and reared at $18^{\circ} \mathrm{C}$. Emerged flies were transferred to $29^{\circ} \mathrm{C}$ and entrained for $3 \mathrm{~d}$ in $12 \mathrm{~h} \mathrm{light} / 12 \mathrm{~h}$ dark (LD), followed by $3 \mathrm{~d}$ in constant darkness (DD). Flies were collected at CT2 and CT14. Cells were dissociated from 
Table 3. Free-running locomotor rhythms ${ }^{a}$

\begin{tabular}{|c|c|c|c|c|c|}
\hline Temperature & Genotype & Period \pm SEM (h) & Power \pm SEM & $n$ & $\% R$ \\
\hline \multirow[t]{20}{*}{$25^{\circ} \mathrm{C}$} & Pdf-GAL4, UAS-dcr2/+ & $24.5 \pm 0.08$ & $101.8 \pm 9.42$ & 32 & 62.5 \\
\hline & Pdf-GAL4, UAS-dcr2/UAS-Rnb RNAi 23099 & $26.6 \pm 0.09^{* * *}$ & $108.9 \pm 7.04$ & 29 & 79.3 \\
\hline & tim-GAL4/+ & $24.4 \pm 0.05$ & $119.2 \pm 5.91$ & 26 & 92.3 \\
\hline & tim-GAL4/+;UAS-Rnb RNAi2 23099 & $26.3 \pm 0.04^{* * *}$ & $136.9 \pm 4.68$ & 32 & 100 \\
\hline & tub-GAL4/+ & $23.7 \pm 0.04$ & $156.8 \pm 8.4$ & 62 & 96.7 \\
\hline & tub-GAL4/UAS-Rnb RNAi ${ }^{23099}$ & $25.2 \pm 0.07^{* * *}$ & $98.5 \pm 8.5$ & 40 & $80.0^{*}$ \\
\hline & Pdf-Gal4/+ & $24.0 \pm 0.06$ & $84.2 \pm 5.0$ & 61 & 95.0 \\
\hline & UAS-RnB::Venus/+ & $23.6 \pm 0.04$ & $94.3 \pm 6.2$ & 57 & 96.5 \\
\hline & Pdf-GAL4, UAS-Rnb::Venus & $24.1 \pm 0.07$ & $78.9 \pm 5.7$ & 61 & 85.3 \\
\hline & UAS-Rnb RNAi $22573 /+$ & $23.9 \pm 0.06$ & $122.4 \pm 9.8$ & 63 & 87.3 \\
\hline & Pdf-GAL4/UAS-Rnb RNAi $i^{22573}$ & $24.5 \pm 0.02^{* * * *}$ & $174.9 \pm 4.9$ & 122 & 96.7 \\
\hline & $w^{1118}$ & $23.7 \pm 0.17$ & $111.5 \pm 7.20$ & 63 & 93.6 \\
\hline & $R n b^{f 04616} /+$ & $24.0 \pm 0.21$ & $59.9 \pm 3.11$ & 64 & $70.3^{* * *}$ \\
\hline & $R n b^{f 04616}$ & $25.2 \pm 0.76$ & $18.8 \pm 2.25$ & 89 & $14.6^{* * *}$ \\
\hline & $R n b^{f 04616} / D f$ & $23.5 \pm 0.21$ & $80.5 \pm 36.99$ & 16 & $18.8^{* * *}$ \\
\hline & $\mathrm{Rnb}^{f 05400} /+$ & $23.8 \pm 0.9$ & $60.2 \pm 2.59$ & 64 & $67.2^{* * *}$ \\
\hline & $R n b^{f 05400}$ & $25.8 \pm 0.76^{*}$ & $22.6 \pm 3.17$ & 90 & $17.8^{* * *}$ \\
\hline & $R n b^{f 05400} / D f$ & $23.2 \pm 0.2$ & $64.5 \pm 10.53$ & 63 & $23.8^{* * *}$ \\
\hline & $R n b^{f 04616} / R n b^{f 05400}$ & $23.3 \pm 0.1$ & $111.8 \pm 23.9$ & 31 & $35.5^{* * *}$ \\
\hline & $D f /+$ & $23.4 \pm 0.1$ & $74.9 \pm 12.20$ & 10 & $50.0^{* * *}$ \\
\hline $18^{\circ} \mathrm{C} \rightarrow 29^{\circ} \mathrm{C}$ & $w^{1118}$ & $23.7 \pm 0.08$ & $147.1 \pm 9.69$ & 32 & 84 \\
\hline \multirow[t]{6}{*}{ Adult-only } & Pdf-GAL4/+;tub-GAL80ts/+ & $24.3 \pm 0.06$ & $175.7 \pm 6.40$ & 62 & 95.2 \\
\hline & gal1118/ tub-GAL80ts & $23.5 \pm 0.04$ & $134.0 \pm 6.89$ & 58 & 89.7 \\
\hline & UAS-Rnb RNAi ${ }^{23099} /$ Pdf-GAL4; tub-GAL80ts/+ & $26.0 \pm 0.07^{* * *}$ & $109.8 \pm 6.90$ & 31 & 100 \\
\hline & UAS-Rnb RNAi ${ }^{23099} /+;$ gal1118/tub-GAL80ts/+ & $25.4 \pm 0.19^{* * *}$ & $87.3 \pm 7.46$ & 28 & 85.7 \\
\hline & UAS-miR-unf/+;gal1118/ tub-GAL80ts & $25.4 \pm 0.15^{* * *}$ & $127.5 \pm 5.70$ & 87 & 97.7 \\
\hline & UAS-miR-unf/UAS-Rnb RNAi23099; gal1118/tub-GAL80ts & $26.1 \pm 0.11^{* * *}$ & $118.6 \pm 5.15$ & 61 & 93.4 \\
\hline $29^{\circ} \mathrm{C} \rightarrow 18^{\circ} \mathrm{C}$ & Pdf-GAL4/+;tub-GAL80ts/+ & $24.1 \pm 0.06$ & $94.1 \pm 6.93$ & 62 & 80.6 \\
\hline Developmental & UAS-Rnb RNAii ${ }^{23099} / P d f-G A L 4 ;$ tub-GAL80ts/+ & $24.4 \pm 0.08^{* *}$ & $93.3 \pm 7.33$ & 31 & 93.5 \\
\hline
\end{tabular}

${ }^{a} n$, Number of flies; \%R, \% of rhythmic flies. Rhythmicity was compared with the driver control or with control genotypes ( $w^{1118}$ for Rnb mutants) by a $\chi^{2}$ test.

Periods were compared with the controls (Student's t test): ${ }^{*} p<0.05$; $^{* *} p<0.01$; ${ }^{* *} p<0.001 ;{ }^{* * *} p<0.0001$.

\begin{abstract}
$\sim 70$ brains as previously described (Nagoshi et al., 2010), except brains were treated with papain for $25 \mathrm{~min}$. After trituration in $300 \mu \mathrm{l} \mathrm{SM}$ active medium containing $50 \mu \mathrm{M}$ AP5, $20 \mu \mathrm{M}$ DNQX, $0.1 \mu \mathrm{M}$ TTX, the cell suspension was filtered through a sterile $70 \mu \mathrm{m}$ membrane and distributed to 3 wells of 3 two-well chamber glass slides (LabTek II, Nunc) kept on ice. The second well of each slide contained ice to cool the cells while we sorted GFP-positive cells on a fluorescence microscope equipped with a motorized stage and a needle holder (CellSorter Company for Innovations). Cells were picked manually using capillaries with $50 \mu \mathrm{m}$ tip diameter. Collected cells were released in a clean part of the chamber and immediately picked up again, greatly reducing the amount of debris, before cells were transferred to a fresh slide in $100 \mu$ l medium spread in a $1 \times 1 \mathrm{~cm}$ GeneFrame (ThermoFisher Scientific). Cells were reselected for 2 rounds and finally transferred using a new capillary with $30 \mu \mathrm{m}$ tip diameter to $100 \mu \mathrm{l}$ lysis buffer (Absolutely RNA Nanoprep Kit; Agilent). Cells of multiple collections were pooled if needed to obtain $\sim 200$ cells per sample, and total RNA was extracted according to manufacturer's instructions. RNA was converted to cDNA and amplified using the SMART-Seq4 Kit (Takara Bio). Libraries were generated using NexteraXT (Illumina) and sequenced on an Illumina HiSeq 2500 or 4000.
\end{abstract}

\section{Results}

\section{Identification of UNF direct targets}

We identified UNF direct targets by Chromatin-IP tiling array (ChIP-chip) using anti-UNF antibodies (Lin et al., 2009). Because UNF levels cycle in the s-LNvs with a peak around ZT2 $(2 \mathrm{~h}$ after light-on in LD cycles) (Beuchle et al., 2012), ChIP assays were performed using adult heads harvested at ZT2 or ZT14 in LD. Fifty-five significant binding sites above a stringent $p<10^{-7}$ cutoff were identified. Nine UNF binding sites were found within $1 \mathrm{~kb}$ of the previously identified CLK-binding peaks (Abruzzi et al., 2011), suggesting that UNF regulates the transcription of some CLK/CYC target genes (Fig. 1A). More UNF binding peaks were identified at ZT14 than at ZT2, which is the opposite trend to the UNF accumulation in the LNvs. This finding suggests that UNF is more transcriptionally active and has a higher turnover rate at ZT14 than at ZT2, consistent with the previous UNF ChIP results in S2 cells (Jaumouillé et al., 2015) (Table 1).

UNF is expressed in the 9 PDF-positive LNvs and in the mushroom body (MB), which consists of $\sim 2000$ neurons per hemisphere (Lin et al., 2009; Sung et al., 2009). No significant binding peaks ( $>5 \%$ false discovery rate, $p<10^{-7}$ ) were identified in the flies with MB-specific unf knockdown with D52H-Gal4 (Aso et al., 2009). Therefore, not surprisingly, the ChIP data largely reflect the UNF targets in the MB. Some of the targets may be shared between MB neurons and LNvs, but the LNvs-specific sites were undetectable in this assay. This explains why no UNFbinding peaks above background levels were detected in the per gene (Jaumouillé et al., 2015).

\section{CG7837 $(R n b)$ is a target of UNF and controls behavioral rhythms in the s-LNv master pacemaker neurons}

To examine whether any of the UNF direct target genes is involved in the clockwork or output mechanism of the LNvs, we next performed a behavior screen for locomotor activity, in which candidate genes were constitutively knocked down in the LNvs with Pdf-GAL4. We selected 39 UNF target genes for screening, considering the rank and the expression levels of their transcripts in the s-LNvs (Kula-Eversole et al., 2010). For each candidate gene, one or two UAS-RNAi lines from the VDRC libraries were used, avoiding those with off-target sites. Fly loco- 
A

$29^{\circ} \mathrm{C} \rightarrow 18^{\circ} \mathrm{C} \quad$ Knockdown during development
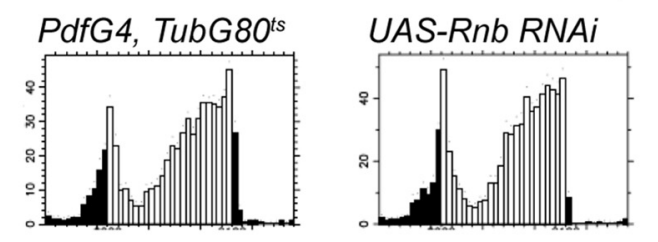

Pdf, Tub80 > Rnb RNAi
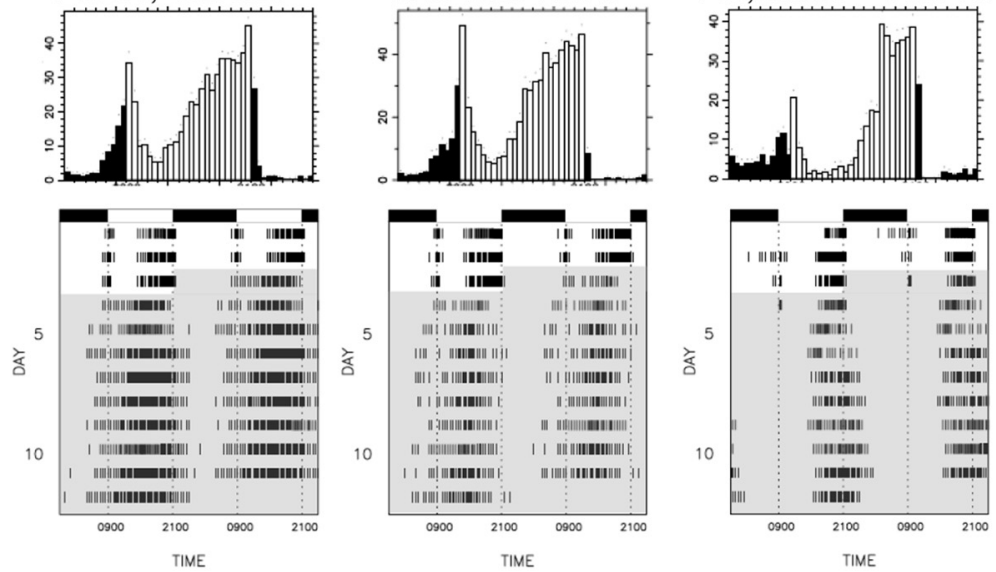

B

$18^{\circ} \mathrm{C} \rightarrow 29^{\circ} \mathrm{C}$ Adult-specific knockdown
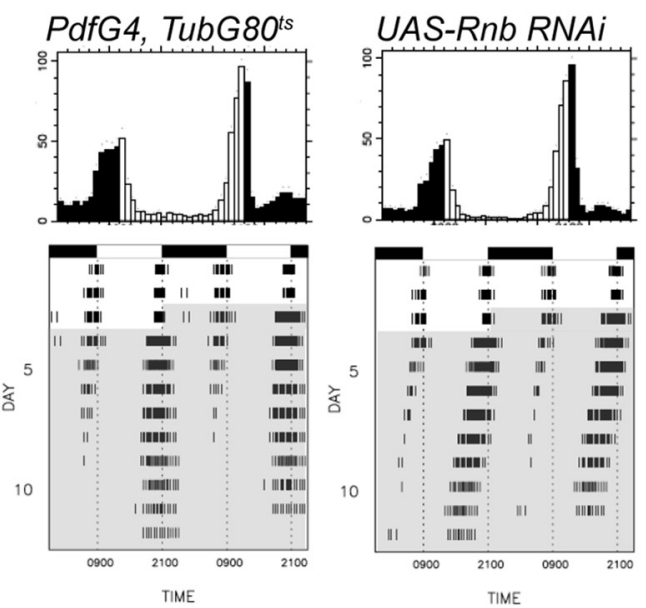

Pdf, Tub80 > Rnb RNAi

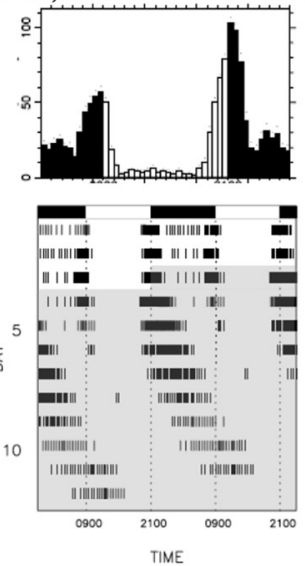

C

CTO

CT4

Cont.
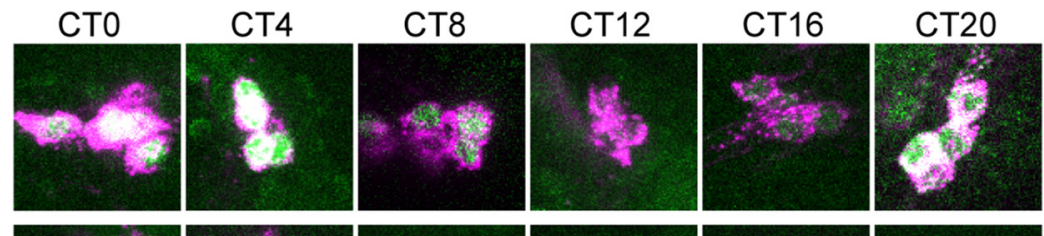

Rnb KD
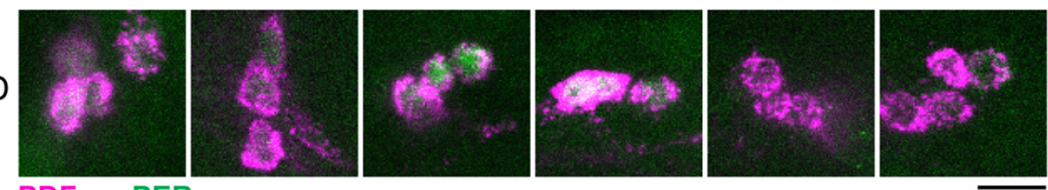

D
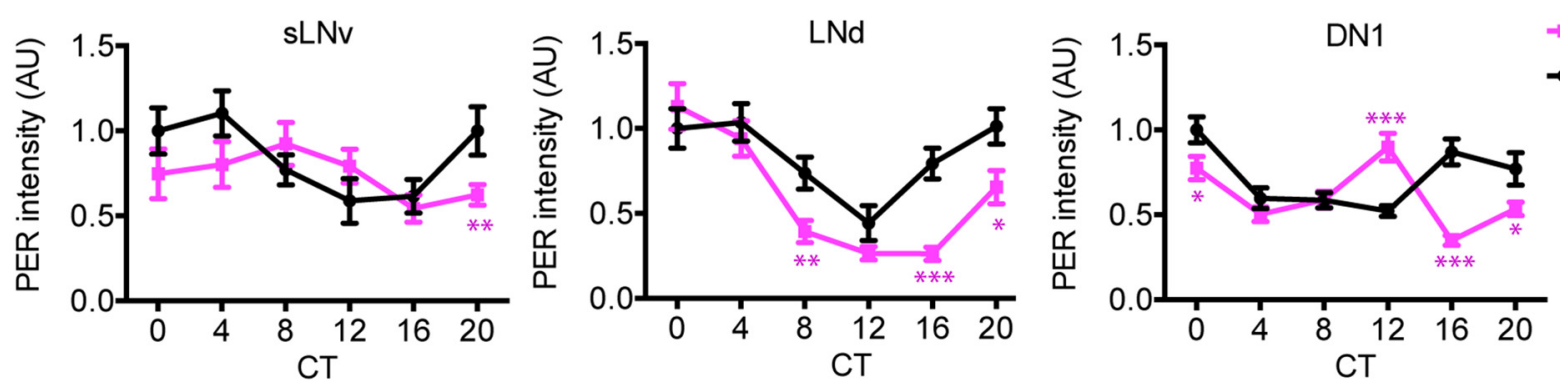

Figure 2. Rnb is required in adult $L$ Nvs for the generation of high-amplitude molecular rhythms and $24 \mathrm{~h}$ locomotor rhythms. $A, B, R n b$ was conditionally knocked down in the $L$ Nvs by expressing

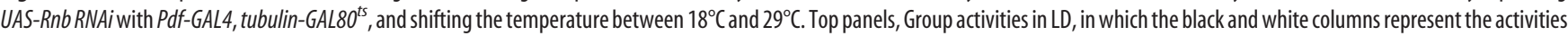
in the night and day, respectively. Bottom panels, Double-plotted actograms show the average locomotor activities of groups of flies in LD (white background) (Figure legend continues.) 
motor behavior was assayed in standard $12 \mathrm{~h} / 12 \mathrm{~h} \mathrm{LD}$ cycles followed by DD at $25^{\circ} \mathrm{C}$.

None of the knockdowns significantly reduced the freerunning rhythm strength; however, several knockdowns altered the period length (Table 2 ; Fig. 1B). We focused subsequent studies on the CG7837 gene, as its knockdown resulted in the longest period among the genes tested $(25.6 \pm 0.03 \mathrm{~h}$ with VDRC RNAi line \#23099). Coexpression of dicer 2 (dcr2) with CG7837 RNAito increase RNAi efficacy further lengthened the period by $\sim 1 \mathrm{~h}$ $(26.6 \pm 0.09$ h). CG7837 knockdown in pan-clock neurons by tim-GAL4 also resulted in long-period rhythms. In addition, knockdown of CG7837 in the LNvs with a second RNAi line (VDRC \#22573) also lengthened the period $(24.5 \pm 0.03 \mathrm{~h})$ (Table 3). We used VDRC \#23099 line for subsequent studies as it knocks down CG7837 more efficiently than the line \#22573 (Fig. 3A).

Furthermore, CG7837 mRNA levels were significantly reduced in the heads of the trans-heterozygous combination of two unf hypomorphic mutants, unf $f^{x / z 0001}$ (Bates et al., 2010) (Fig. $1 C$ ), indicating that UNF positively regulates CG7837 gene expression. RNAseq of isolated LNvs verified that both unf and CG7837 are expressed in the LNvs (Fig. 1D). Together with the fact that period lengthening by LNv-targeted CG7837 knockdown is similar to the phenotype of UNF adult-specific knockdown in the LNvs (Beuchle et al., 2012), these results suggest that CG7837 acts downstream of UNF in the LNvs and contributes to the regulation of circadian behavior.

CG7837 encodes a 975-amino acid long protein of unknown function containing N-terminal Armadillo (ARM)-repeats and a C-terminal BTB/POZ domain. CG7837 mRNA is broadly and nonrhythmically expressed in the brain (Kula-Eversole et al., 2010; Nagoshi et al., 2010). Because of its role in the rhythms and the structure containing Armadillo and $B \mathrm{~TB}$, we named this gene $R$ and $B(R n b)$.

\section{$R n b$ acts downstream of UNF in adulthood in the s-LNvs}

$\mathrm{UNF}$ is required for the functional development of the s-LNvs and for the functioning of adult s-LNvs as the master circadian pacemaker (Beuchle et al., 2012). To determine whether Rnb acts downstream of UNF during development or in adulthood, we conditionally knocked down Rnb in the LNvs using a combination of the temperature-dependent repressor of GAL4 (tubulin-Galso $\left.{ }^{t_{s}}\right)$ and an LNv-specific GAL4 driver (Pdf-GAL4 or gal1118-GAL4) (McGuire et al., 2004). Rnb RNAi expression was silenced when flies were maintained at the permissive temperature of GAL80 $0^{\text {ts }}$ $\left(18^{\circ} \mathrm{C}\right)$ and induced at the GAL80 ${ }^{\text {ts }}$ restrictive temperature $\left(29^{\circ} \mathrm{C}\right)$. Unlike the unf developmental knockdown, Rnb knockdown during development only mildly lengthened free-running behavioral period of adult flies without affecting the rhythm strength (Table 3; Fig. 2A). In contrast, the adult-restricted knockdown of $R n b$ in the LNvs lengthened the free-running pe-

(Figure legend continued.) and DD (gray background). A, Locomotor behavior following Rnb knockdown during development. $\boldsymbol{B}$, Locomotor behavior of the flies with adult-restricted Rnb knockdown. $\boldsymbol{C}, \boldsymbol{D}$, Rnb was knocked down in the LNvs only during adulthood, and the PER levels in the clock neurons were analyzed on DD3. $C$, Representative confocal $z$-stack images of the $\mathrm{s}$-LNvs stained for PER (green) and PDF (magenta). Scale bar, $10 \mu \mathrm{m}$. D, Quantification of the PER staining intensity. PER rhythms are delayed in the s-LNvs of the Rnb knocked down flies compared with control flies (Pdf-GAL4, tub-GAL80 ${ }^{t 5}$ ), and the rhythms in DN1s are impaired. Significant differences in PER levels between the two genotypes at several time points: ${ }^{*} p<$ $0.05,{ }^{* *} p<0.01,{ }^{* * *} p<0.001$ (Student's $t$ test). CT, Circadian time (i.e., subjective time in constant darkness). riod by $\sim 2 \mathrm{~h}$ (Table 3 ; Fig. $2 B$ ). LD behavior was not affected by either of the Rnb knockdowns (Fig. 2A,B).

PER rhythms in the s-LNvs were delayed in DD by the LNvtargeted adult-specific knockdown of $R n b$ (Fig. 2C,D). The rhythms in the DN1s were also impaired non-cell-autonomously, consistent with previous findings that s-LNvs control DN1 rhythms (Nitabach et al., 2006; Zhang et al., 2010a; Beuchle et al., 2012; Jaumouillé et al., 2015) (Fig. 2D). These cellular and behavioral phenotypes associated with the Rnb adult-restricted knockdown in the LNvs are reminiscent of those caused by the unf adult-specific knockdown (Beuchle et al., 2012).

Double knockdown of Rnb and unf in adult LNvs further lengthened the long-period rhythms caused by single knockdowns ( $R n b$ single knockdown vs double knockdown with gal1118, $p<0.05$; unf single vs double knockdown with gal1118, $p<0.001, t$ test) (Table 3 ). This additive effect likely reflects the combination of partial knockdown (Fig. 3A) and the fact that UNF has more functions than controlling Rnb expression, as evident from the ChIP results (Table 1). Together, these results indicate that $R n b$ mediates at least part of the role of UNF in adult $\mathrm{s}$-LNvs in the generation of $24 \mathrm{~h}$ molecular rhythms and circadian behavioral rhythms.

\section{$R n b$ is required for the generation of molecular rhythms in the s-LNvs}

To learn more about the role of $R n b$ in circadian behavioral control, we assayed the behavior of two $R n b$ mutants, $R n b^{f 04616}$ and $R n b^{f 05400}$. Both alleles were caused by a transposon insertion and severely hypomorphic (Fig. 3A). Heterozygous and homozygous mutants of both alleles, and the trans-heterozygotes and hemizygous flies showed significantly reduced free-running locomotor rhythmicity and reduced morning anticipation (Table 3; Fig. 3B). These results confirmed that $R n b$ is required for normal circadian locomotor behavior. Rnb mRNA levels in heterozygous flies were $<50 \%$ of the wild-type levels (Fig. $3 A$ ), which is consistent with the reduced locomotor rhythmicity of the heterozygotes and hemizygotes and indicates that $R n b$ is a haploinsufficient gene. The ubiquitous knockdown of Rnb with UAS-Rnb RNAi $i^{23099}$ driven by tubulin-GAL4, which reduces Rnb mRNA levels in fly heads by $\sim 65 \%$ (Fig. $3 A$ ), lengthened the period and reduced behavioral rhythmicity (Table 3). Because Rnb knockdown in all clock neurons by a pan-clock neuron driver tim-GAL4 also lengthened the period but did not affect the rhythm strength (Table 3), Rnb expressed in nonclock cells likely contributes to reinforcing behavioral rhythm strength.

We next examined whether the Rnb mutation affects the functioning of clock neurons by immunostaining PER and PDF throughout the subjective day. PER levels were markedly reduced during the peak time of PER accumulation (CT22-CT2), and the rhythms were dampened in the s-LNvs of Rnb homozygous mutants on DD3. In the LNds and DN1s, PER oscillations were phase-advanced in $R n b$ mutants, suggesting a slight shortening of free-running rhythms in these cells (Fig. 3C). Considering that mutations more strongly reduce $R n b$ mRNA levels than RNAi (Fig. $3 A$ ), the cellular phenotypes in the s-LNvs were comparable between RNAi and mutations and confirm that the loss of Rnb expression disrupts the molecular clockwork in the s-LNvs. The rhythms of the LNd and DN1s were differently affected between $R n b$ mutations and RNAi (Figs. 2D, 3C). This effect likely reflects the fact that $s-\mathrm{LNv}$ clocks were more severely disrupted by $R n b$ mutations than by RNAi. Consequently, the communication between the s-LNvs and other clock neurons was more profoundly 


\section{A}

CG7837 (Rnb)

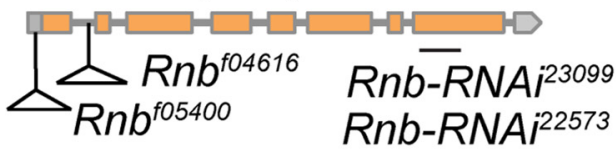

B $W^{1118}$
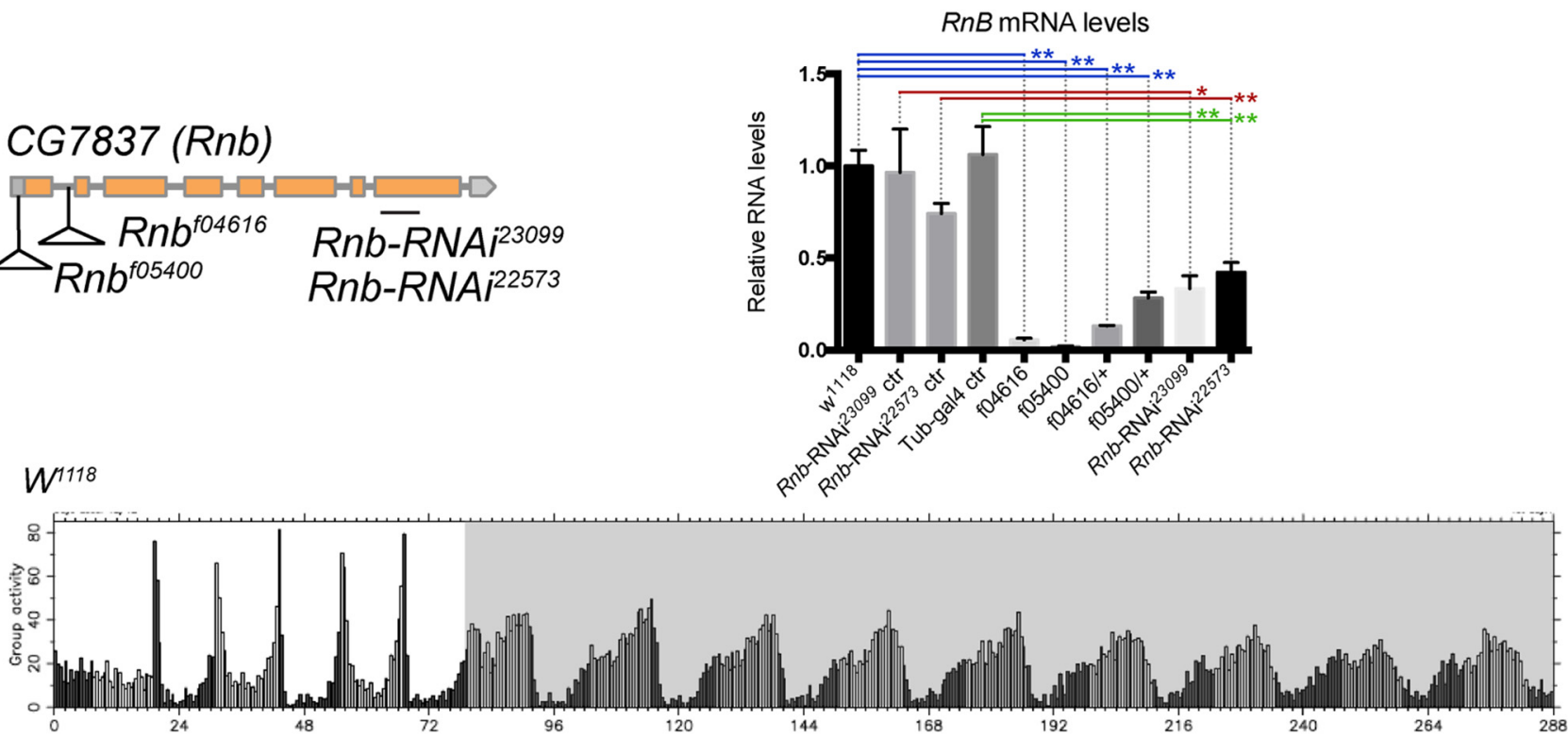

$R n b^{f 04616}$

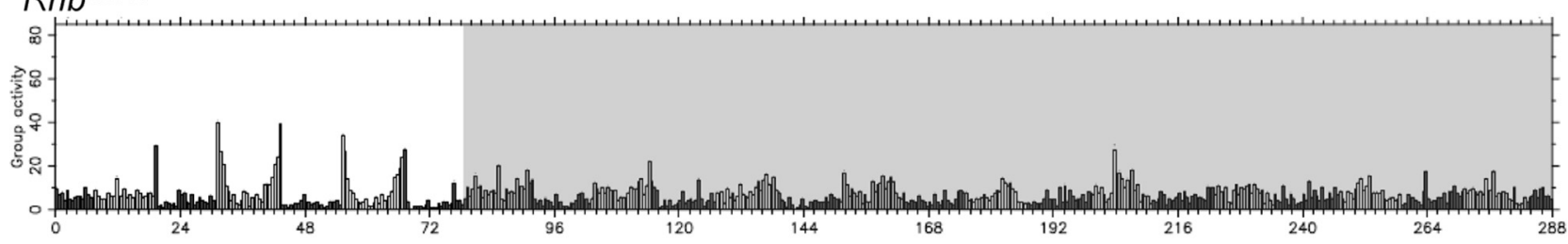

$R n b^{f 05400}$

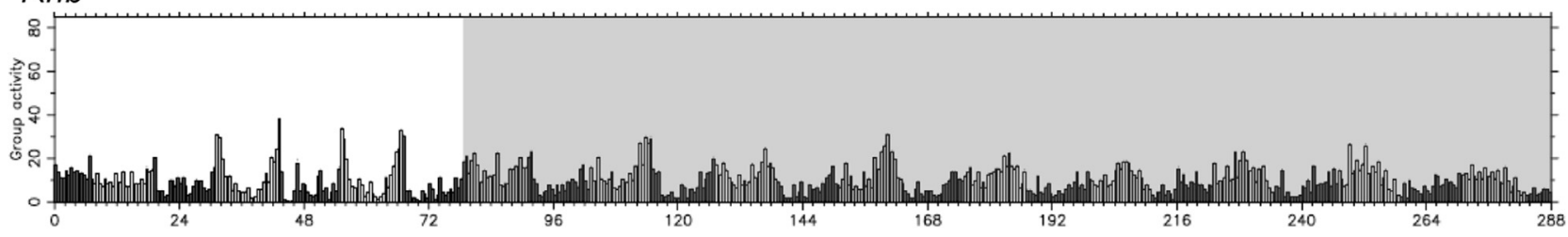

$R n b^{f 04616 / R} R b^{f 05400}$

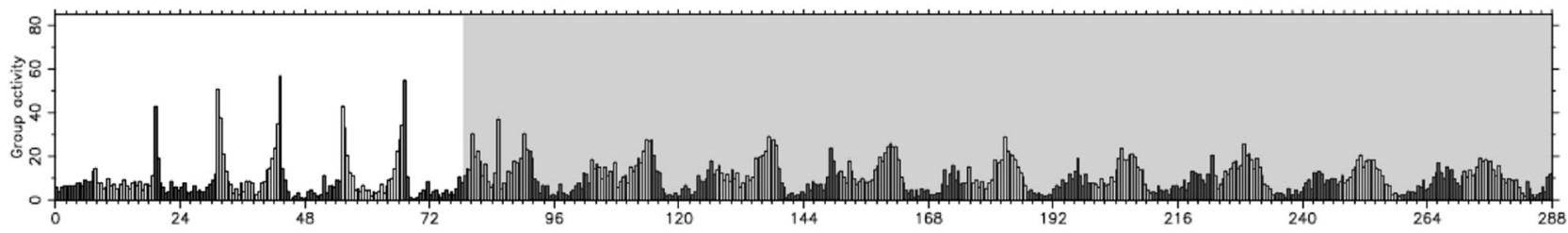

Time (h)

C

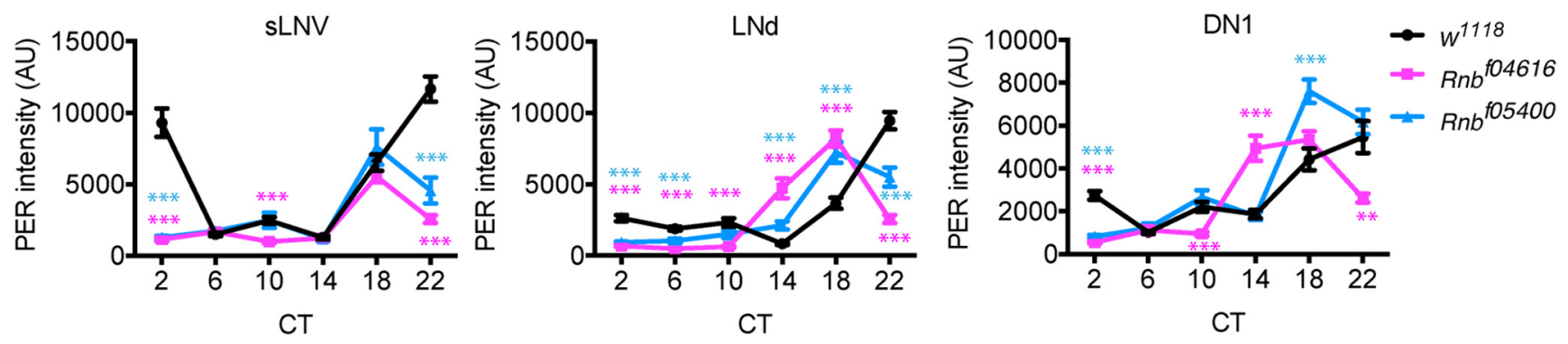

Figure 3. Rnb mutation impairs s-LNv clocks and circadian locomotor behavior. $\boldsymbol{A}$, Left, Rnb gene, mutants, and the Rnb RNAi target site. Right, Rnb mRNA levels at ZT14 in the heads of Rnb mutants, knockdowns (tubulin-GAL4 driving UAS-Rnb RNAi \#23099 or \#22573), and controls relative to those of $w^{1118}$ were analyzed using qPCR. ${ }^{*} p<0.05$, ${ }^{* *} p<0.01$ (Student's $t$ test). $B$, Cartesian plots for group average activities of $w^{1118}$ and $R n b$ mutant flies in LD and DD. C, PER levels of $w^{1118}$ and $R n b$ mutant flies on DD3. PER rhythms were advanced $\sim 4 \mathrm{~h}$ in both $R n b^{f 04616}$ (magenta) and $R n b^{f 05400}$ (blue) mutants in all three cell types, with a profound reduction in rhythm amplitude in the s-LNvs. ${ }^{*} p<0.05,{ }^{* *} p<0.01,{ }^{* * *} p<0.001$ (Student's $t$ test). 
A
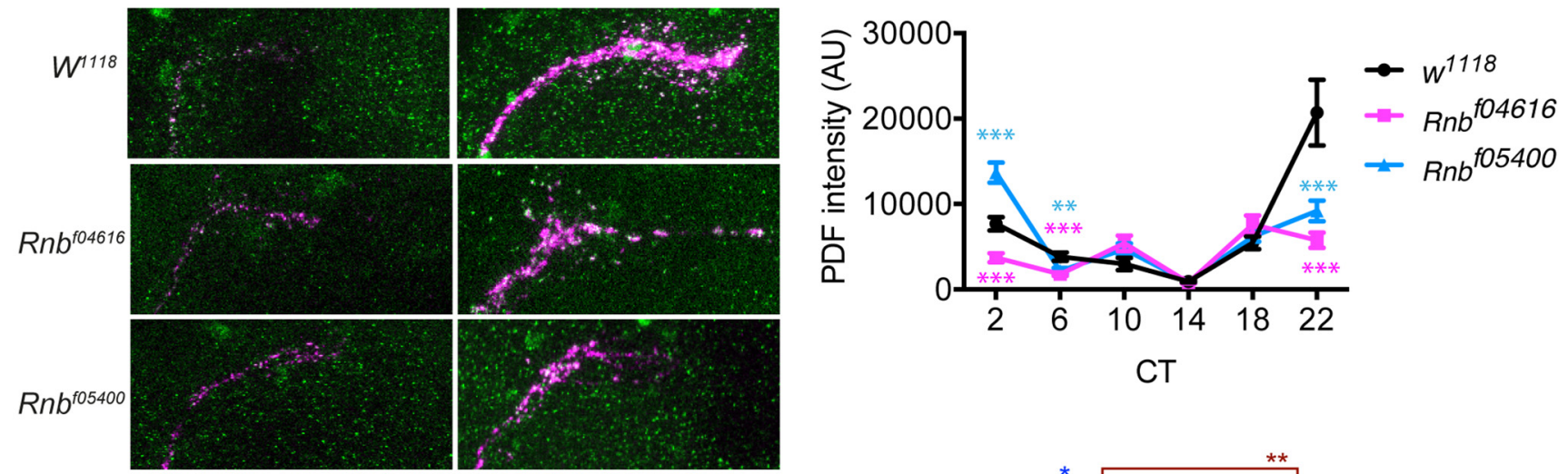

B

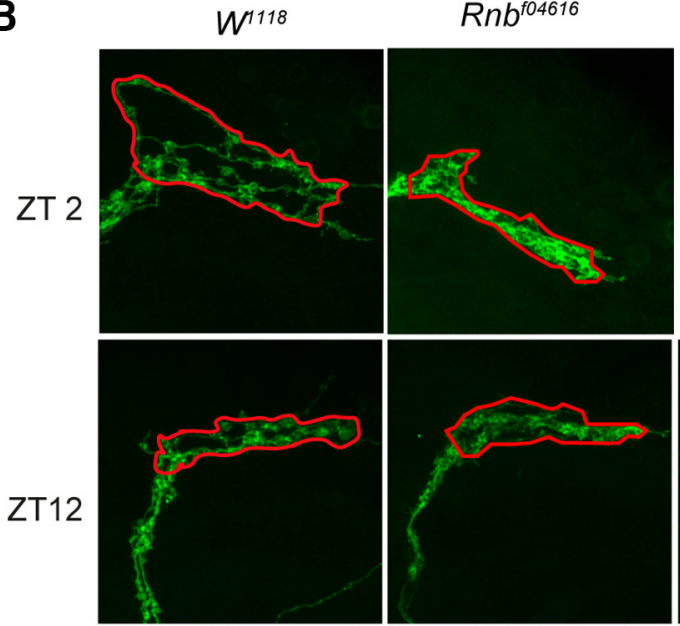

$P d f>m C D 8::$ VENUS

E

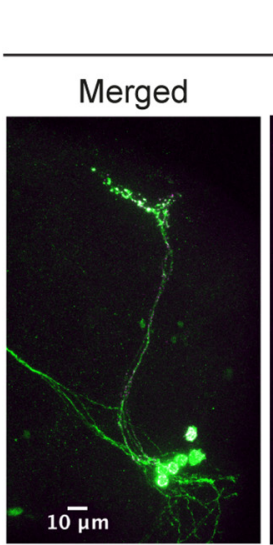

$P d f>G F P$

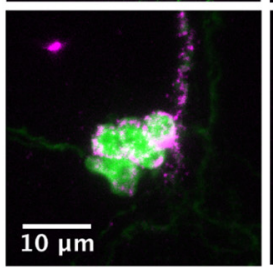

PDF
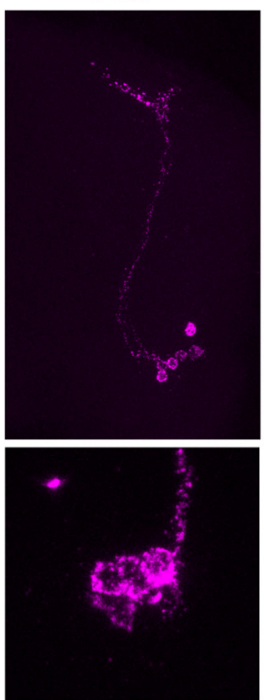

$R n b^{f 05400}$

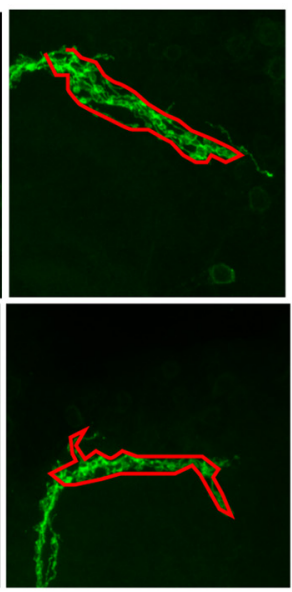

C
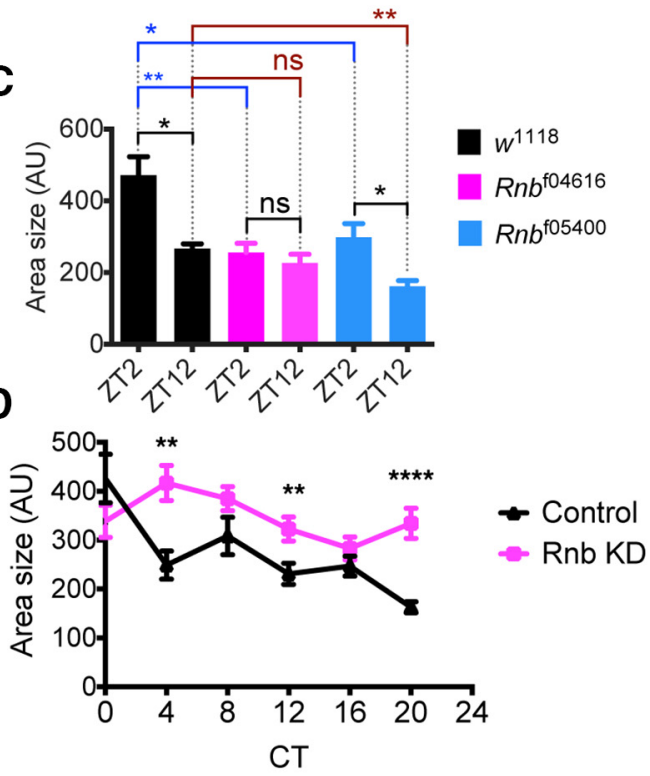

Pdf $>$ Rnb::Venus

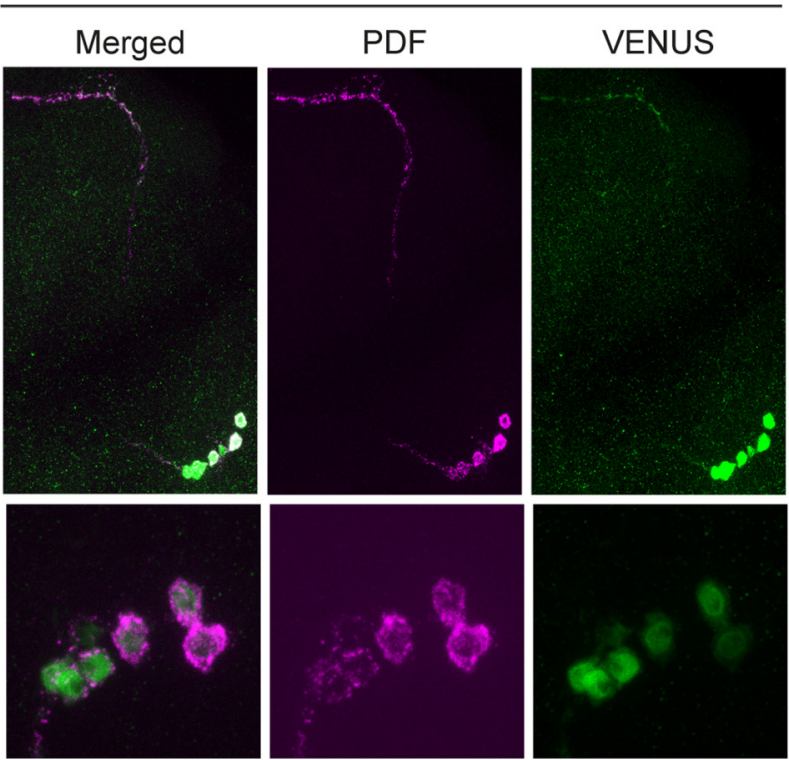

Figure 4. Rnb is required for the output rhythms of the s-LNvs. A, Left, Representative images of anti-PDF signals in the s-LNv dorsal termini in Rnb mutants and $w^{1118}$ on DD3. Right, Quantification of PDF levels on DD3 in the $s$-LNv axon termini. The rhythms of PDF signals in the axon termini of Rnb mutants are dampened compared with those in $w^{1118}$. ${ }^{*} p<0.05$, ${ }^{* *} p<0.01$, ${ }^{* * *} p<0.001$ (Student's t test). B, C, s-LNv axonal arborization in $w^{1118}$ and Rnb mutants was analyzed by expressing UAS-mCD8::Venus with Pdf-GAL4 and staining with anti-GFP antibodies at two time points in LD. $\boldsymbol{B}$, Representative confocal $z$-stack images with the ROIs for quantification of dorsal terminal spread. $\boldsymbol{C}$, Mean area covered by the axonal arborization $\pm S E M .{ }^{*} p<0.05,{ }^{* *} p<$ 0.01 (Student's t test). D, Quantification of s-LNv axonal arborization in the flies with adult-restricted LNv-targeted Rnb knockdown analyzed at six time points on DD3. ${ }^{* *} p<0.01,{ }^{* * * *} p<0.0001$. E, Expression of GFP or Rnb::Venus was driven by Pdf-GAL4 in wild-type flies and analyzed by anti-GFP/PDF staining at ZT2. Images were captured (Figure legend continues.) 
disturbed in the mutants, which provoked the LNds and DN1s to cycle with their intrinsically short period (Yoshii et al., 2009).

Rhythmic PDF accumulation in the axonal termini and circadian reorganization of axonal arbors are the prominent output rhythms of the s-LNvs (Park et al., 2000; Fernández et al., 2008). We found that PDF levels in the dorsal projections of the s-LNvs were reduced during its peak time of accumulation (CT22-CT2) and its rhythms were blunted in Rnb mutants (Fig. 4A). Rnb $b^{f 04616}$ mutants showed a stronger phenotype than $R n b^{f 05400}$, which might reflect the nature of the mutations: $R n b^{f 04616}$ might produce aberrantly spliced variants, which might have a gain-offunction effect on the PDF accumulation rhythms (Fig. 3A).

We also visualized the s-LNv dorsal termini at two time points by expressing a membrane-tagged Venus (mCD8::Venus) (Fig. $4 B$ ). Quantification of the axonal terminal spread showed that s-LNv axonal arborization remained more closed in $R n b$ mutants compared with the control flies (Fig. $4 C$ ). In $R n b^{f 04616}$ mutants, rhythms of the LNv axonal spread were also abolished. The observation that $R n b^{f 04616}$ exhibited stronger phenotype than $R n b^{f 05400}$ is consistent with the effect of these alleles to the rhythms of PDF accumulation (Fig. 4A).

The above findings suggest that $R n b$ contributes to axonal remodeling either by affecting development of the LNvs or by playing a role in axonal remodeling regulation in adult s-LNvs. To test the latter possibility, we knocked down Rnb in the LNvs only during adulthood and analyzed the s-LNv dorsal arborization at 6 time points on DD3. Axonal remodeling rhythms persisted in the knockdown flies with a delayed phase, consistent with the long-period rhythms caused by the Rnb adult-restricted knockdown. However, the rhythm amplitude was reduced because s-LNv axonal arbors remained more open at the baseline state than in control flies (Fig. 4D). These results indicate that $R n b$ is involved in the regulation of the s-LNv axonal remodeling in adulthood. Nevertheless, the effects of Rnb mutations and adult-restricted knockdown are not identical: mutations render axonal arbors more closed, whereas adult-specific knockdown put them in more open state. These differences suggest that loss of function of $R n b$ during development has also important consequences for axonal structural remodeling of adult s-LNvs.

Rhythmic PDF accumulation is indirectly controlled by the molecular clock via an unknown mechanism involving matrix metalloproteinase Mmp1 (Park et al., 2000; Depetris-Chauvin et al., 2014), and PDF abundance correlates with the degree of axonal spread (Depetris-Chauvin et al., 2014). Our results are consistent with these previous findings and suggest that $R n b$ contributes to the control of rhythmic PDF accumulation and axonal remodeling by regulating the molecular clocks. These data also explain why morning anticipation behavior in $\mathrm{LD}$, which requires normal PDF signaling, is markedly reduced in $R n b \mathrm{mu}-$ tants (Fig. 3B).

To gain insight into the mechanisms by which Rnb contributes to the functioning of the s-LNvs, we sought to examine the subcellular localization of RNB protein. To this end, we drove the expression of Rnb::Venus fusion gene in the LNvs by Pdf-GAL4. These flies showed normal locomotor rhythms (Table 1). Whereas UAS-GFP driven by Pdf-GAL4 was present throughout the cell body and projections of the s-LNvs, by using the same

(Figure legend continued.) using the same microscopy setting for both genotypes. Representative images of the s-LNvs (top) and high-magnification views of the s-LNv cell bodies (bottom) stained for GFP or VENUS (green) and PDF (magenta).

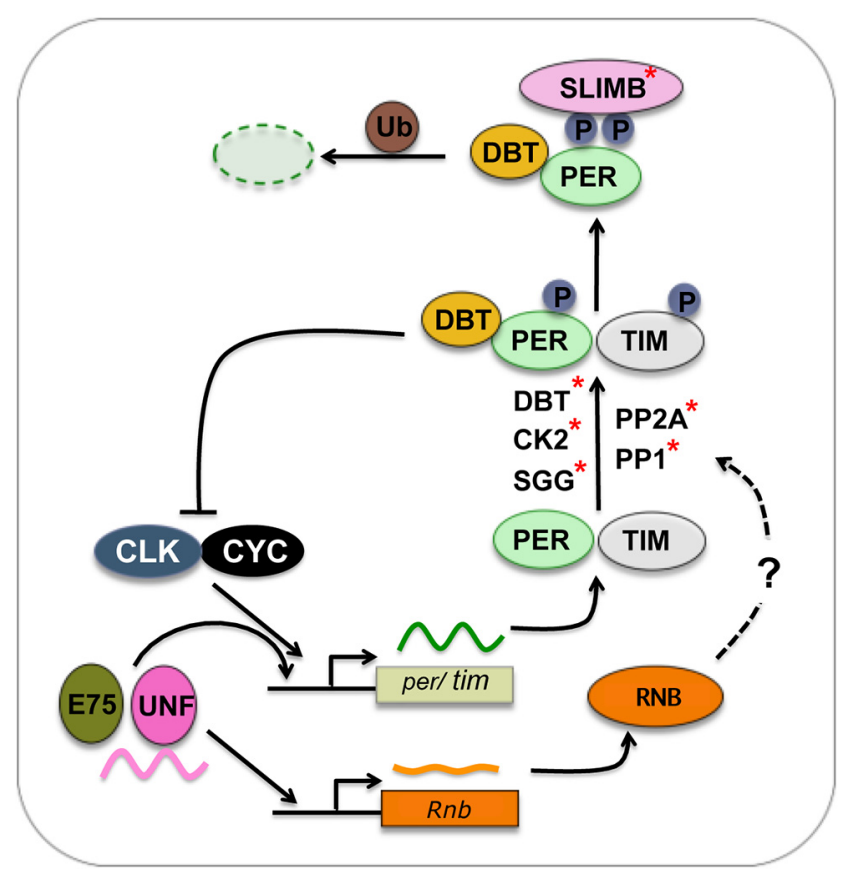

Figure 5. A model for the role of RNB in the s-LNv master pacemaker neurons. In the s-LNvs, UNF and E75 cooperatively enhance the transcription of per. UNF also binds to the Rnb gene and promotes its expression. RNB interacts with the molecular clock feedback loops to reinforce molecular oscillations and output rhythms. The role of RNB might be mediated by some of the molecular clock components (*) that are also involved in the Wnt/Wingless signaling pathway.

microscopy setting, the RNB::VENUS signal was detected almost exclusively in the cell body with enrichment in the nucleus (Fig. $4 E)$. RNB has a theoretical molecular weight of $110.7 \mathrm{kDa}$; and, particularly as a fusion with VENUS $(\sim 27 \mathrm{kDa})$, it is unlikely to passively diffuse into the nucleus (Wang and Brattain, 2007). Although overexpression of tagged protein may not fully recapitulate the subcellular localization of endogenous protein, these results at least suggest that RNB can be actively transported into the nucleus to execute its function in the molecular clock.

\section{Discussion}

Recent studies have shown the existence of the clock regulatory machineries that distinguish s-LNvs master pacemaker neurons from other clock neuron subpopulations (Lee et al., 2016; Top et al., 2016). In this study, to gain mechanistic insight into the differential regulation of the molecular clock, we analyzed the downstream pathway of UNF, an s-LNv-specific molecular clock regulator (Beuchle et al., 2012; Jaumouillé et al., 2015). We identified $R n b$ as a direct target of UNF and demonstrated that $R n b$ acts downstream of UNF in the s-LNvs to control molecular clockwork and behavioral output. These results establish that UNF interacts with the core TTFL in the s-LNvs via at least two pathways: regulation of per transcription (Jaumouillé et al., 2015) and the upregulation of Rnb expression (Fig. 5). Together, these results corroborate the emerging view that molecular clocks are regulated differently within the pacemaker circuit. This is accomplished by the cell-type specific genetic and biochemical environments, underpinning the functional differences among the pacemaker neuron subgroups.

UNF ChIP-chip experiments identified 55 high-confidence binding sites, of which 9 sites were cotargets of CLK (Table 1). CLK is expressed in all clock-containing cells, whereas UNF expression is restricted to the LNvs; thus, these sites may not be cobound by CLK and UNF within the LNvs. Notably, one of the 
CLK/UNF cotargets, Ecdysone receptor $(E c R)$ is an upstream regulator of the nuclear receptor E75 (Gauhar et al., 2009). Because E75 and UNF coregulate per transcription in the s-LNvs to reinforce TTFL (Jaumouillé et al., 2015), coregulation of EcR expression by CLK and UNF would add an additional feedback loop in the s-LNvs clockwork. However, the knockdown of the $E c R$ (CG1765) gene by Pdf-GAL4 did not alter behavioral rhythms, and this possibility remains an open question. Increasing RNAi efficacy by coexpressing $d c r 2$ and $E c R$ gain-of-function experiments would provide a more clear-cut answer to this question.

$R n b$ loss-of-function mutations dampen the molecular rhythms, reduce PDF levels, blunt PDF accumulation rhythms, and perturb rhythms in axonal remodeling in the s-LNvs. Concomitantly, Rnb mutants have reduced morning anticipation in LD and are arrhythmic in DD (Figs. 3, 4). Adult-specific LNvtargeted $\mathrm{Rnb}$ knockdown disrupts s-LNv molecular clocks, dampens s-LNv axonal remodeling rhythms, and lengthens freerunning rhythms (Figs. 2, 4D). It is noteworthy that PDF levels at the s-LNv dorsal terminal appeared moderately reduced by unf knockdown in the LNvs; however, the reduction was statistically nonsignificant (data not shown). This result probably reflects insufficient decrease of $R n b$ level but is nevertheless consistent with UNF being an upstream regulator of $R n b$. In addition, although $R n b$ knockdown in developing or adult LNvs did not impair morning anticipatory behavior (Fig. $2 A, B$ ), probably also due to insufficient knockdown (Fig. $3 A$ ), these results together indicate that $R n b$ expression within the s-LNvs during adulthood is required for the pacemaking role of the s-LNvs.

RNB contains an ARM-repeat and a BTB/POZ domain. The $\mathrm{BTB} / \mathrm{POZ}$ domain mediates protein-protein interaction and is often found in transcriptional regulators (Collins et al., 2001). Indeed, a recent study using an elegant assay in S2 cells showed that RNB has transcriptional cofactor activity (Stampfel et al., 2015). Nuclear localization of overexpressed RNB::VENUS (Fig. $4 E$ ) suggests that RNB can be actively transported to the nucleus and supports the possibility that RNB is involved in the transcriptional control of the molecular clock in the pacemaker neurons.

ARM-repeat proteins are critical members of the Wnt/Wingless signaling pathway, which controls a wide range of developmental and cell biological processes (Willert and Jones, 2006; Bejsovec, 2013). Interestingly, RNB was identified as a potential regulator of the Wnt/Wingless signaling pathway in two prior genetic screens (DasGupta et al., 2005; Gersten et al., 2014). Intriguingly, several members of the Wnt/Wingless pathway are essential regulators of the fly TTFL (Rosato et al., 2006). Therefore, RNB might also function at the interface between the Wnt/ Wingless signaling pathway and the molecular clock in the generation of molecular rhythms and output of the s-LNvs (Fig. 5). Our study provides an entry point to future research projects aimed at the detailed molecular dissection of the mechanisms by which RNB affects circadian functions in the s-LNv master pacemaker neurons.

\section{References}

Abruzzi KC, Rodriguez J, Menet JS, Desrochers J, Zadina A, Luo W, Tkachev S, Rosbash M (2011) Drosophila CLOCK target gene characterization: implications for circadian tissue-specific gene expression. Genes Dev 25: 2374-2386. CrossRef Medline

Abruzzi K, Chen X, Nagoshi E, Zadina A, Rosbash M (2015) RNA-seq profiling of small numbers of Drosophila neurons. Methods Enzymol 551: 369-386. CrossRef Medline

Aso Y, Grübel K, Busch S, Friedrich AB, Siwanowicz I, Tanimoto H (2009) The mushroom body of adult Drosophila characterized by GAL4 drivers. J Neurogenet 23:156-172. CrossRef Medline
Bates KE, Sung CS, Robinow S (2010) The unfulfilled gene is required for the development of mushroom body neuropil in Drosophila. Neural Dev 5:4. CrossRef Medline

Bejsovec A (2013) Wingless/Wnt signaling in Drosophila: the pattern and the pathway. Mol Reprod Dev 80:882-894. CrossRef Medline

Beuchle D, Jaumouillé E, Nagoshi E (2012) The nuclear receptor unfulfilled is required for free-running clocks in Drosophila pacemaker neurons. Curr Biol 22:1221-1227. CrossRef Medline

Blanchardon E, Grima B, Klarsfeld A, Chélot E, Hardin PE, Préat T, Rouyer F (2001) Defining the role of Drosophila lateral neurons in the control of circadian rhythms in motor activity and eclosion by targeted genetic ablation and PERIOD protein overexpression. Eur J Neurosci 13:871-888. CrossRef Medline

Cavanaugh DJ, Geratowski JD, Wooltorton JR, Spaethling JM, Hector CE, Zheng X, Johnson EC, Eberwine JH, Sehgal A (2014) Identification of a circadian output circuit for rest: activity rhythms in Drosophila. Cell 157: 689-701. CrossRef Medline

Collins T, Stone JR, Williams AJ (2001) All in the family: the BTB/POZ, KRAB, and SCAN domains. Mol Cell Biol 21:3609-3615. CrossRef Medline

DasGupta R, Kaykas A, Moon RT, Perrimon N (2005) Functional genomic analysis of the Wnt-wingless signaling pathway. Science 308:826-833. CrossRef Medline

Depetris-Chauvin A, Fernández-Gamba A, Gorostiza EA, Herrero A, Castaño EM, Ceriani MF (2014) Mmpl processing of the PDF neuropeptide regulates circadian structural plasticity of pacemaker neurons. Plos Genet 10:e1004700. CrossRef Medline

Fernández MP, Berni J, Ceriani MF (2008) Circadian remodeling of neuronal circuits involved in rhythmic behavior. PLoS Biol 6:e69. CrossRef Medline

Gauhar Z, Sun LV, Hua S, Mason CE, Fuchs F, Li TR, Boutros M, White KP (2009) Genomic mapping of binding regions for the Ecdysone receptor protein complex. Genome Res 19:1006-1013. CrossRef Medline

Gersten M, Zhou D, Azad P, Haddad GG, Subramaniam S (2014) Wnt pathway activation increases hypoxia tolerance during development. PLoS One 9:e103292. CrossRef Medline

Hardin PE (2011) Molecular genetic analysis of circadian timekeeping in Drosophila. Adv Genet 74:141-173. CrossRef Medline

Hardin PE, Panda S (2013) Circadian timekeeping and output mechanisms in animals. Curr Opin Neurobiol 23:724-731. CrossRef Medline

Jaumouillé E, Machado Almeida P, Stähli P, Koch R, Nagoshi E (2015) Transcriptional regulation via nuclear receptor crosstalk required for the Drosophila circadian clock. Curr Biol 25:1502-1508. CrossRef Medline

Johnson WE, Li W, Meyer CA, Gottardo R, Carroll JS, Brown M, Liu XS (2006) Model-based analysis of tiling-arrays for ChIP-chip. Proc Natl Acad Sci U S A 103:12457-12462. CrossRef Medline

Kula-Eversole E, Nagoshi E, Shang Y, Rodriguez J, Allada R, Rosbash M (2010) Surprising gene expression patterns within and between PDFcontaining circadian neurons in Drosophila. Proc Natl Acad Sci U S A 107:13497-13502. CrossRef Medline

Lee E, Cho E, Kang DH, Jeong EH, Chen Z, Yoo SH, Kim EY (2016) Pacemaker-neuron-dependent disturbance of the molecular clockwork by a Drosophila CLOCK mutant homologous to the mouse Clock mutation. Proc Natl Acad Sci U S A 113:904-913. CrossRef Medline

Lim C, Allada R (2013) Emerging roles for post-transcriptional regulation in circadian clocks. Nat Neurosci 16:1544-1550. CrossRef Medline

Lin S, Huang Y, Lee T (2009) Nuclear receptor unfulfilled regulates axonal guidance and cell identity of Drosophila mushroom body neurons. PLoS One 4:e8392. CrossRef Medline

McGuire SE, Mao Z, Davis RL (2004) Spatiotemporal gene expression targeting with the TARGET and gene-switch systems in Drosophila. Sci STKE 2004:pl6. CrossRef Medline

Menet JS, Abruzzi KC, Desrochers J, Rodriguez J, Rosbash M (2010) Dynamic PER repression mechanisms in the Drosophila circadian clock: from on-DNA to off-DNA. Genes Dev 24:358-367. CrossRef Medline

Mohawk JA, Green CB, Takahashi JS (2012) Central and peripheral circadian clocks in mammals. Annu Rev Neurosci 35:445-462. CrossRef Medline

Nagoshi E, Sugino K, Kula E, Okazaki E, Tachibana T, Nelson S, Rosbash M (2010) Dissecting differential gene expression within the circadian neuronal circuit of Drosophila. Nat Neurosci 13:60-68. CrossRef Medline

Nitabach MN, Wu Y, Sheeba V, Lemon WC, Strumbos J, Zelensky PK, White 
BH, Holmes TC (2006) Electrical hyperexcitation of lateral ventral pacemaker neurons desynchronizes downstream circadian oscillators in the fly circadian circuit and induces multiple behavioral periods. J Neurosci 26:479-489. CrossRef Medline

Park JH, Helfrich-Förster C, Lee G, Liu L, Rosbash M, Hall JC (2000) Differential regulation of circadian pacemaker output by separate clock genes in Drosophila. Proc Natl Acad Sci U S A 97:3608-3613. CrossRef Medline

Peschel N, Helfrich-Förster C (2011) Setting the clock- by nature: circadian rhythm in the fruitfly Drosophila melanogaster. FEBS Lett 585:1435-1442. CrossRef Medline

Renn SC, Park JH, Rosbash M, Hall JC, Taghert PH (1999) A pdf neuropeptide gene mutation and ablation of PDF neurons each cause severe abnormalities of behavioral circadian rhythms in Drosophila. Cell 99:791-802. CrossRef Medline

Rosato E, Tauber E, Kyriacou CP (2006) Molecular genetics of the fruit-fly circadian clock. Eur J Hum Genet 14:729-738. CrossRef Medline

Shafer OT, Rosbash M, Truman JW (2002) Sequential nuclear accumulation of the clock proteins period and timeless in the pacemaker neurons of Drosophila melanogaster. J Neurosci 22:5946-5954. Medline

Stampfel G, Kazmar T, Frank O, Wienerroither S, Reiter F, Stark A (2015) Transcriptional regulators form diverse groups with context-dependent regulatory functions. Nature 528:147-151. CrossRef Medline

Stoleru D, Peng Y, Nawathean P, Rosbash M (2005) A resetting signal between Drosophila pacemakers synchronizes morning and evening activity. Nature 438:238-242. CrossRef Medline

Sung C, Wong LE, Chang Sen LQ, Nguyen E, Lazaga N, Ganzer G, McNabb SL, Robinow S (2009) The unfulfilled/DHR51 gene of Drosophila mela- nogaster modulates wing expansion and fertility. Dev Dyn 238:171-182. CrossRef Medline

Top D, Harms E, Syed S, Adams EL, Saez L (2016) GSK-3 and CK2 kinases converge on timeless to regulate the master clock. Cell Rep 16:357-367. CrossRef Medline

Wang JW, Beck ES, McCabe BD (2012) A modular toolset for recombination transgenesis and neurogenetic analysis of Drosophila. PLoS One 7:e42102. CrossRef Medline

Wang R, Brattain MG (2007) The maximal size of protein to diffuse through the nuclear pore is larger than $60 \mathrm{kDa}$. FEBS Lett 581:3164-3170. CrossRef Medline

Willert K, Jones KA (2006) Wnt signaling: is the party in the nucleus? Genes Dev 20:1394-1404. CrossRef Medline

Yao Z, Shafer OT (2014) The Drosophila circadian clock is a variably coupled network of multiple peptidergic units. Science 343:1516-1520. CrossRef Medline

Yoshii T, Wülbeck C, Sehadova H, Veleri S, Bichler D, Stanewsky R, HelfrichFörster C (2009) The neuropeptide pigment-dispersing factor adjusts period and phase of Drosophila's clock. J Neurosci 29:2597-2610. CrossRef Medline

Zhang L, Chung BY, Lear BC, Kilman VL, Liu Y, Mahesh G, Meissner RA, Hardin PE, Allada R (2010a) DN1(p) circadian neurons coordinate acute light and PDF inputs to produce robust daily behavior in Drosophila. Curr Biol 20:591-599. CrossRef Medline

Zhang Y, Liu Y, Bilodeau-Wentworth D, Hardin PE, Emery P (2010b) Light and temperature control the contribution of specific DN1 neurons to Drosophila circadian behavior. Curr Biol 20:600-605. CrossRef Medline 